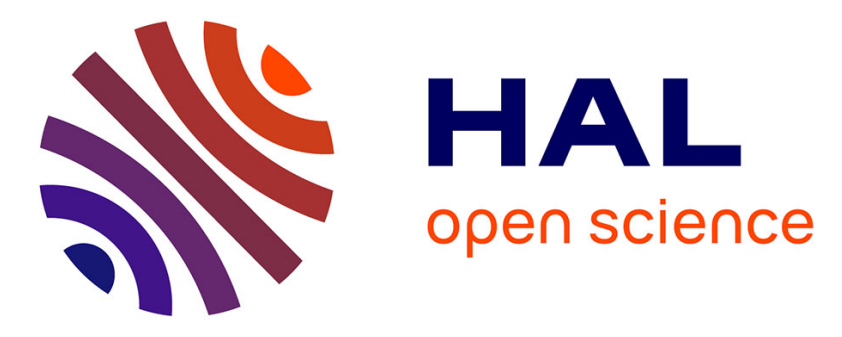

\title{
Computational homogenization of nano-materials accounting for size effects via surface elasticity
}

Ali Javili, Georges Chatzigeorgiou, Andrew T. Mcbride, Paul Steinmann, Christian Linder

\section{- To cite this version:}

Ali Javili, Georges Chatzigeorgiou, Andrew T. Mcbride, Paul Steinmann, Christian Linder. Computational homogenization of nano-materials accounting for size effects via surface elasticity. GAMMMitteilungen, 2015, 38 (2), pp.285-312. 10.1002/gamm.201510016 . hal-01515306

\section{HAL Id: hal-01515306 https://hal.univ-lorraine.fr/hal-01515306}

Submitted on 8 Apr 2020

HAL is a multi-disciplinary open access archive for the deposit and dissemination of scientific research documents, whether they are published or not. The documents may come from teaching and research institutions in France or abroad, or from public or private research centers.
L'archive ouverte pluridisciplinaire HAL, est destinée au dépôt et à la diffusion de documents scientifiques de niveau recherche, publiés ou non, émanant des établissements d'enseignement et de recherche français ou étrangers, des laboratoires publics ou privés. 


\title{
Computational homogenization of nano-materials accounting for size effects via surface elasticity
}

\author{
Ali Javili ${ }^{1, *}$, George Chatzigeorgiou ${ }^{2}$, Andrew T. McBride ${ }^{3}$, Paul Steinmann ${ }^{4}$, \\ and Christian Linder ${ }^{1}$ \\ ${ }^{1}$ Department of Civil and Environmental Engineering, Stanford University, 473 Via Ortega, \\ Stanford, CA 94305, USA \\ ${ }^{2}$ LEM3-UMR 7239 CNRS, Arts et Métiers ParisTech, 4 Rue Augustin Fresnel, 57078 Metz, \\ France \\ ${ }^{3}$ Centre for Research in Computational and Applied Mechanics, University of Cape Town, \\ 5th floor, Menzies Building, Private Bag X3, 7701 Rondebosch, South Africa \\ ${ }^{4}$ Chair of Applied Mechanics, University of Erlangen-Nuremberg, Egerlandstr. 5, 91058 \\ Erlangen, Germany
}

Key words Homogenization, Surface elasticity, Size effects, Nano-materials

The objective of this contribution is to establish a first-order computational homogenization framework for micro-to-macro transitions of porous media that accounts for the size effects through the consideration of surface elasticity at the microscale. Although the classical (firstorder) homogenization schemes are well established, they are not capable of capturing the well-known size effects in nano-porous materials. In this contribution we introduce surface elasticity as a remedy to account for size effects within a first-order homogenization scheme. This proposition is based on the fact that surfaces are no longer negligible at small scales.

Following a standard first-order homogenization ansatz on the microscopic motion in terms of the macroscopic motion, a Hill-type averaging condition is used to link the two scales. The averaging theorems are revisited and generalized to account for surfaces. In the absence of surface energy this generalized framework reduces to classical homogenization. The influence of the length scale is elucidated via a series of numerical examples performed using the finite element method. The numerical results are compared against the analytical ones at small strains for tetragonal and hexagonal microstructures. Furthermore, numerical results at small strains are compared with those at finite strains for both microstructures. Finally, it is shown that there exists an upper bound for the material response of nano-porous media. This finding surprisingly restricts the notion of "smaller is stronger".

\section{Introduction}

During the last three decades a significant research interest on nano-structured materials has been developed, such as carbon nanotubes [1], nano-channel-array materials [2], nanobeams [3], nano-wires [4], nano-crystalline metals [5], nano-belts [6] and thin films [7], with their size in the order of a few to hundreds of nanometers. The significant electrical, thermal 
or mechanical response of nano-materials is due to their dominant surface effects and the fact that the surface-to-volume ratio is proportional to the inverse of the dimension and hence, very large at the nano-scale.

Surface effects can be modeled using various continuum approaches. The study of the behavior of surfaces dates back to the works of Laplace, Young and Gibbs, see e.g. the reviews by Duan et al. [8] and Javili et al. [9] for further details. A phenomenological model that accounts for surfaces with their own constitutive behavior by considering tensorial surface stresses has been proposed by Gurtin and Murdoch [10] stemming from the work by Scrive [11]. Central to the theory of surface elasticity is that the surface is energetic in the sense that it possesses its own mechanical and constitutive structures which are, in general, independent of those associated with the bulk. More details on surface elasticity theory and variants thereof can be found in [12-17]. The overall response of continua due to the presence of surfaces with their own constitutive law has been extensively investigated using the surface elasticity theory, see e.g. [18-21]. Computational aspects of surface elasticity theory using the finite element method are detailed in [22-24].

If one needs to identify the overall response of a composite with energetic surfaces in its microstructure, then the developed phenomenological models for surfaces should be combined with micromechanics and homogenization. The main principles of homogenization were introduced by Hill [25] and Hill and Rice [26] as a consistent methodology to link the macroscopic and microscopic scales. Homogenization forms the basis for computational micro-to-macro transition and is a powerful tool to understand the response of heterogenous materials. For further details on computational homogenization, see e.g. [27-47] and references therein. Methods for obtaining effective properties for materials that contain energetic surfaces have been developed recently using analytical homogenization [48-52].

This contribution deals with computational homogenization of porous materials accounting for the surface elasticity of the pores. This works bears certain similarities to [53], however, it provides a more elaborate study and answers several questions raised recently. The key features and objectives of this manuscript are:

- to investigate the effect of pore-to-volume and surface-to-volume ratios in the presence of surface elasticity,

- to compare tetragonal and hexagonal micro-structures,

- to study the response of the material with and without surface effects,

- to compare small strain with finite strain response,

- to compare analytical solutions with numerical results (in the case of small strains),

- to show the bounded response of nano-porous materials from both analytical and numerical observations.

\section{Structure of this manuscript}

This manuscript is organized as follows. Notation and definitions are shortly introduced. Section 2 deals with theoretical aspects of computational homogenization at finite strains accounting for surfaces. The balance equations at the macroscale and the microscale are briefly 
introduced and modified averaging theorems are proposed to link the two scales. The modified averaging theorems reduce to the classical ones in the absence of surfaces. Also, the classical Hill-Mandel condition is generalized to consistently establish an incremental energy equivalence between the scales. Section 3 elucidates the developed theory by a series of numerical examples. A well established analytical estimate for this type of problems is given and its validity is assessed using numerical examples based on the finite element method. Numerical simulations are carried out for various sizes, volume fractions, load cases and deformations, and also for both tetragonal and hexagonal microstructures. Section 4 concludes this work, discusses possible extensions and gives an outlook.

\section{Notation and definitions}

Direct notation is adopted throughout. At the microscale, quantities defined on the energetic surface are distinguished from those in the bulk material by a hat placed above the quantity. That is, $\{\hat{\bullet}\}$ refers to a surface variable with its bulk counterpart being $\{\bullet\}$. Moreover, macroscopic quantities are differentiated from microscale quantities by the left super-script "M" placed next to the quantity. That is, $\mathrm{M}\{\bullet\}$ refers to a macroscopic variable with its microscopic counterpart being $\{\bullet\}$.

The terms "macro" and "micro" are frequently used instead of macroscopic and microscopic, respectively. In the discussion of the results, the terms "stronger" and "weaker" are frequently used instead of "stiffer" and "softer", respectively. This choice is made to compare the material behavior with the familiar notion of "smaller is stronger" and should not be confused with the classical definitions of these terms in mechanics.

Within the classical homogenization context, the term "size" usually refers to the size of the window zoomed at the material microstructure. When this window is large enough such that an apparent macroscopic property of interest converges, then the window becomes a representative volume element abbreviated as "RVE" and its size defines the "RVE size", see for instance [54] for further details on the definition of the RVE.

First-order computational homogenization is missing a length-scale or physical size of the RVE and therefore, "size" as defined above does not introduce any confusion. This is not the case here. In this manuscript the terminology "size" and in particular "RVE size" is used somewhat differently from its conventional meaning in computational homogenization, but closer to its meaning in the general sense of (physical) size. In particular, we consider perfectly periodic microstructures such that the unit cell is by definition representative and hence "RVE". In the discussions of the numerical results varying the "RVE size" indicates varying the physical size or dimension of the "RVE" such that a size effect is observed.

\section{Theoretical framework of homogenization}

Consider a porous material where the pores are enclosed within material surfaces with a constitutive response different to that of the bulk material. We assume throughout this work that the common assumption of length-scale separation associated with homogenization holds. Therefore, the macroscopic problem is large enough compared to the length scale associated with the microscale problem. In this contribution, all relations are represented only in the Lagrangian description, keeping in mind that it is straightforward to formulate the problem in the Eulerian description. Recall that the primary objective of this work is to account for 
size effects within a computational homogenization scheme through the consideration of surfaces. Therefore, we limit the discussion to major relations and definitions that are essential for the paper to be self-contained. Further details on computational homogenization and its underlying assumptions can be found in the extensive references listed in the introduction.

\subsection{Macroscale}

Consider a continuum body that takes the material configuration at time $t=0$ and the spatial configuration at any time $t>0$, as shown in Fig. 1. At the macro level the body occupies the domain ${ }^{\mathrm{M}} \mathcal{B}_{0}$ with the boundary $\partial^{\mathrm{M}} \mathcal{B}_{0}$ at time $t=0$ with outward surface unit normal ${ }^{\mathrm{M}} \boldsymbol{N}$. The macroscopic spatial configuration is denoted ${ }^{\mathrm{M}} \mathcal{B}_{t}$ with the boundary $\partial^{\mathrm{M}} \mathcal{B}_{t}$ and the surface unit normal ${ }^{\mathrm{M}} \boldsymbol{n}$. A material point in the macroscale is characterized by a position vector ${ }^{\mathrm{M}} \boldsymbol{X}$ and is mapped to its spatial counterpart ${ }^{\mathrm{M}} \boldsymbol{x}$ via the non-linear deformation map ${ }^{\mathrm{M}} \boldsymbol{\varphi}$, i.e. ${ }^{\mathrm{M}} \boldsymbol{x}={ }^{\mathrm{M}} \boldsymbol{\varphi}\left({ }^{\mathrm{M}} \boldsymbol{X}\right)$. The corresponding macro deformation gradient ${ }^{\mathrm{M}} \boldsymbol{F}$ maps (linearly) the line element $\mathrm{d}^{\mathrm{M}} \boldsymbol{X}$ in the material configuration to the spatial line element $\mathrm{d}^{\mathrm{M}} \boldsymbol{x}$ according to

$$
\mathrm{d}^{\mathrm{M}} \boldsymbol{x}={ }^{\mathrm{M}} \boldsymbol{F} \cdot \mathrm{d}^{\mathrm{M}} \boldsymbol{X} \quad \text { and } \quad{ }^{\mathrm{M}} \boldsymbol{F}={ }^{\mathrm{M}} \operatorname{Grad}^{\mathrm{M}} \boldsymbol{\varphi} \quad \text { with } \quad{ }^{\mathrm{M}} \mathrm{Grad}\{\bullet\}=\frac{\partial\{\bullet\}}{\partial{ }^{\mathrm{M}} \boldsymbol{X}} .
$$

The governing equations are balances of linear momentum and angular momentum. In the absence of inertial effects, the balance of linear momentum reads

$$
{ }^{\mathrm{M}} \mathrm{Div}^{\mathrm{M}} \boldsymbol{P}+{ }^{\mathrm{M}} \boldsymbol{b}_{0}^{\mathrm{p}}=\mathbf{0} \quad \text { in }{ }^{\mathrm{M}} \mathcal{B}_{0} \quad \text { subject to } \quad{ }^{\mathrm{M}} \boldsymbol{P} \cdot{ }^{\mathrm{M}} \boldsymbol{N}-{ }^{\mathrm{M}} \boldsymbol{t}_{0}^{\mathrm{p}}=\mathbf{0} \quad \text { on } \partial^{\mathrm{M}} \mathcal{B}_{0}^{\mathrm{N}},
$$

with ${ }^{\mathrm{M}} \boldsymbol{b}_{0}^{\mathrm{p}}$ as the macroscopic body force density in the material configuration and ${ }^{\mathrm{M}} \boldsymbol{P}$ the macro Piola stress. The prescribed traction per unit reference area in the material configuration is denoted ${ }^{\mathrm{M}} \boldsymbol{t}_{0}^{\mathrm{p}}$ and is applied on the Neumann boundary $\partial^{\mathrm{M}} \mathcal{B}_{0}^{\mathrm{N}}$. The local form of balance of angular momentum in the material configuration is

$$
{ }^{\mathrm{M}} \boldsymbol{P} \cdot{ }^{\mathrm{M}} \boldsymbol{F}^{\mathrm{t}}={ }^{\mathrm{M}} \boldsymbol{F} \cdot{ }^{\mathrm{M}} \boldsymbol{P}^{\mathrm{t}} .
$$

\subsection{Microscale}

The material configuration at the microscale is denoted $\mathcal{B}_{0}$ and is assumed to be statistically representative of the material. The configuration $\mathcal{B}_{0}$ defines the representative volume element (RVE) in the material configuration and its external boundary is denoted $\partial \mathcal{B}_{0}$ with outward unit normal $\boldsymbol{N}$, see Fig. 2. The spatial configuration at the microscale is defined analogously. The internal boundary of the RVE is the union of the surfaces of the pores within $\mathcal{B}_{0}$ and are collectively denoted as $\mathcal{S}_{0}$ with its spatial counterpart denoted $\mathcal{S}_{t}$. The surfaces of the pores are assumed to be smooth. The outward unit normal to $\mathcal{S}_{0}$ is denoted $\boldsymbol{N}$ and the domain surrounded by $\mathcal{S}_{0}$ is denoted $\mathcal{V}_{0}$ with its volume indicating the pore volume.

Remark: If the external boundary $\partial \mathcal{B}_{0}$ intersects with a pore, the intersection of $\mathcal{S}_{0}$ and $\partial \mathcal{B}_{0}$ forms a curve $\mathcal{C}_{0}:=\partial \mathcal{S}_{0}$ as the boundary of the surface of the pore. The normal to this curve and tangent to $\mathcal{S}_{0}$ is denoted $\widehat{\boldsymbol{N}}$. In this manuscript, we assume that the pores are entirely enclosed within the RVE and the external boundary of the RVE is chosen such that it has no intersection with the surface $\mathcal{S}_{0}$ and therefore $\partial \mathcal{S}_{0}=\emptyset$. This assumption only simplifies the derivations and does not influence the fundamental concepts. 


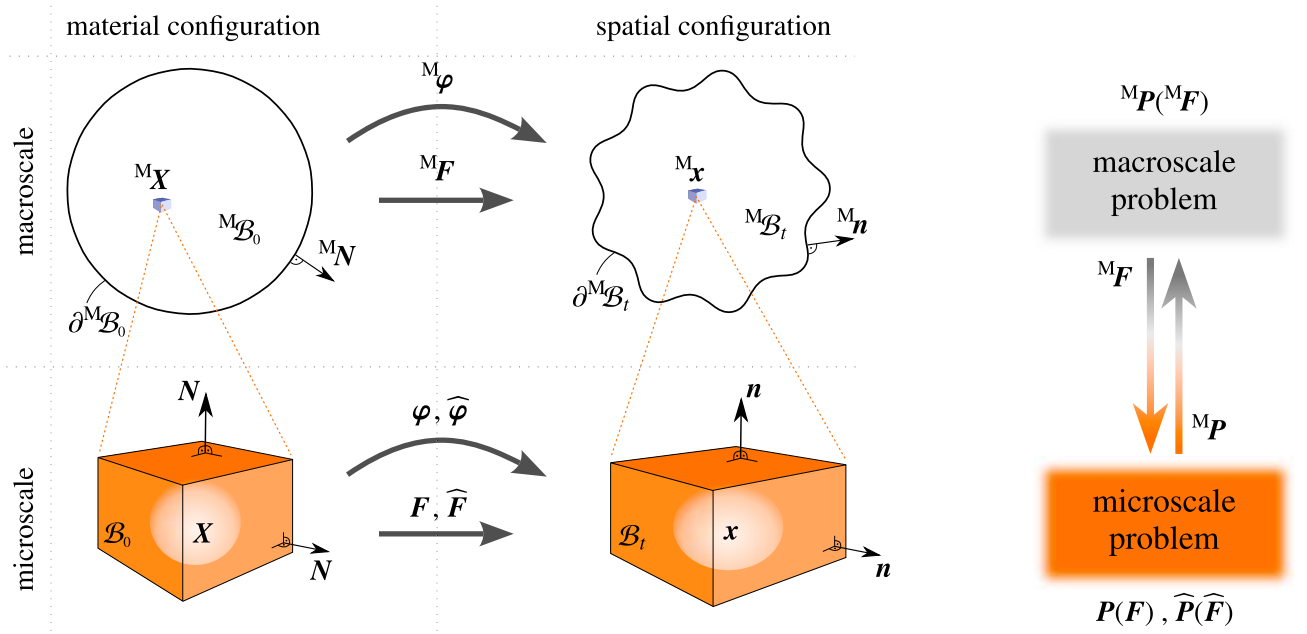

Fig. 1 (online colour at: www.gamm-mitteilungen.org) A graphical summary of computational homogenization. A macroscopic domain ${ }^{\mathrm{M}_{\mathcal{B}_{0}}}$ in the material configuration that maps to the spatial configuration ${ }^{\mathrm{M}} \mathcal{B}_{t}$ via the non-linear deformation map ${ }^{\mathrm{M}} \boldsymbol{\varphi}$. The domain $\mathcal{B}_{0}$ corresponds to a RVE. The motion $\varphi$ of the microscopic RVE is associated with a macroscopic point ${ }^{\mathrm{M}} \boldsymbol{X}$ within the bulk. Constitutive laws at the micro-scale are known and the goal is to compute implicitly the macro stress from the response of the underlying microstructure.

Classical continuum mechanics assigns the free energy only to the bulk material. The same holds for available homogenization schemes. Surface elasticity theory, though, requires additionally an independent surface energy assigned to the surfaces of the pores $\mathcal{S}_{0}$. In this work at the micro level we employ the surface enhanced continuum theory while having the classical continuum theory at the macro level. This is motivated by the fact that due to the small dimensions and, consequently, large area to volume ratio of the micro problem, the surface effects are not negligible at the microscale, however, they can be neglected at the macro level. The free energy density $\Psi$ and the surface free energy density $\widehat{\Psi}$ are assigned to the bulk and to the surfaces of the pores at the microscale, respectively, as shown in Fig. 2.

In a near-identical fashion to the macroscopic problem, the kinematics of the micro problem are now presented. Let $\boldsymbol{X}$ be the position vector of a point in $\mathcal{B}_{0}$ that is mapped via the non-linear deformation map to its counterpart $\boldsymbol{x}$ in the spatial configuration $\mathcal{B}_{t}$. The points on the material surface $\widehat{\boldsymbol{X}}:=\left.\boldsymbol{X}\right|_{\mathcal{S}_{0}}$ are mapped to $\widehat{\boldsymbol{x}}:=\left.\boldsymbol{x}\right|_{\mathcal{S}_{t}}$ via $\widehat{\varphi}$ and since the surface is material, $\widehat{\varphi}=\left.\boldsymbol{\varphi}\right|_{\mathcal{S}_{0}}$. The material line elements $\mathrm{d} \boldsymbol{X} \in \mathcal{B}_{0}$ are mapped to $\mathrm{d} \boldsymbol{x} \in \mathcal{B}_{t}$ via the linear deformation map $\boldsymbol{F}=\operatorname{Grad} \boldsymbol{\varphi}$ with $\operatorname{Grad}\{\bullet\}=\partial\{\bullet\} / \partial \boldsymbol{X}$. On the surface $\mathcal{S}_{0}$ the material line elements $\mathrm{d} \widehat{\boldsymbol{X}}$ are mapped to the spatial (surface) line elements $\mathrm{d} \widehat{\boldsymbol{x}}$ via the surface deformation gradient $\widehat{\boldsymbol{F}}$ as

$$
\mathrm{d} \widehat{\boldsymbol{x}}=\widehat{\boldsymbol{F}} \cdot \mathrm{d} \widehat{\boldsymbol{X}} \quad \text { and } \quad \widehat{\boldsymbol{F}}=\widehat{\operatorname{Grad}} \widehat{\boldsymbol{\varphi}} \quad \text { with } \quad \widehat{\operatorname{Grad}}\{\bullet\}=\frac{\partial\{\bullet\}}{\partial \widehat{\boldsymbol{X}}}=\operatorname{Grad}\{\bullet\} \cdot \widehat{\boldsymbol{I}},
$$

where $\widehat{\boldsymbol{I}}=\boldsymbol{I}-\boldsymbol{N} \otimes \boldsymbol{N}$ denotes the surface identity tensor or rather the surface projection. 

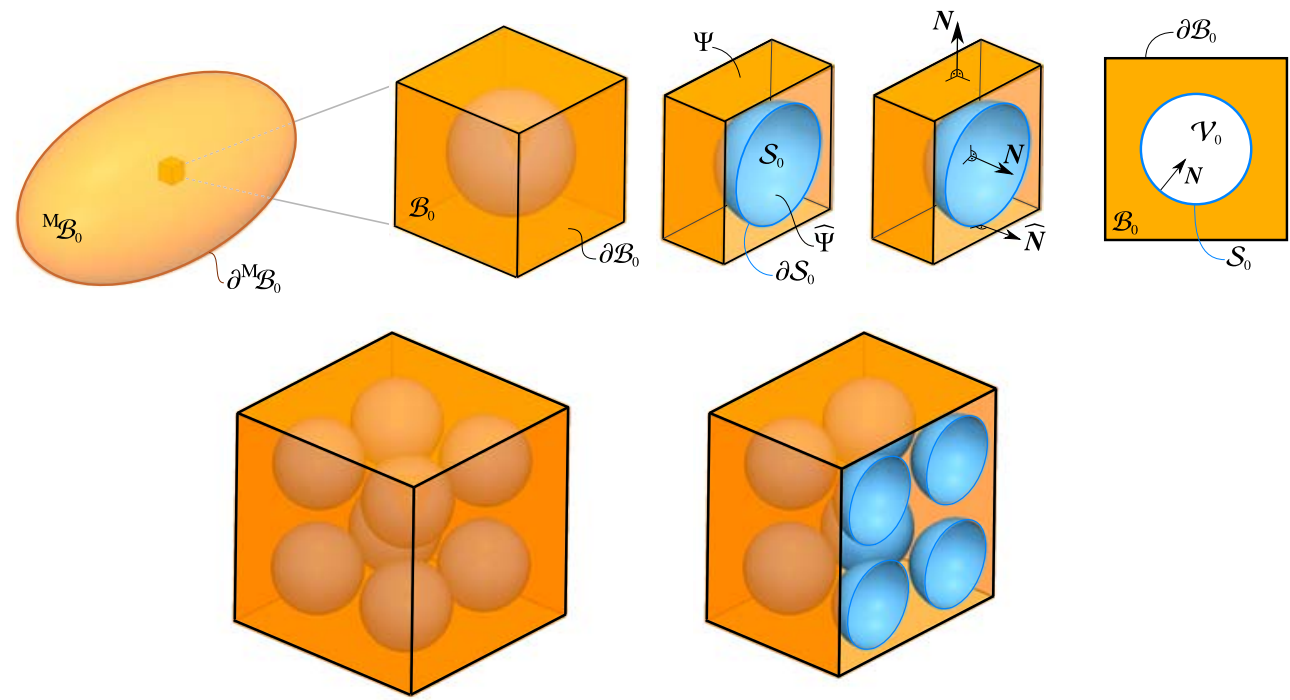

Fig. 2 (online colour at: www.gamm-mitteilungen.org) Porous composite with material surface in the microstructure, separating pores from the bulk material in the material configuration with its twodimensional illustration. Similar to the classical homogenization the constitutive response of the bulk material is described by its free energy $\Psi$. In contrast to the classical homogenization the surface of the pores are also endowed with their free energy $\widehat{\Psi}$ resulting in the size-effect missing in the classical first-order schemes. The external and internal boundary of the RVE are denoted $\partial \mathcal{B}_{0}$ and $\mathcal{S}_{0}$, respectively.

For the microscale problem of interest, the governing equations are balances of linear and angular momentum in the bulk and on the surface. Balance of linear momentum in the bulk reads

$$
\operatorname{Div} \boldsymbol{P}=\mathbf{0} \quad \text { in } \mathcal{B}_{0}
$$

subject to

$$
\boldsymbol{P} \cdot \boldsymbol{N}-\boldsymbol{t}_{0}^{\mathrm{p}}=\mathbf{0} \quad \text { on } \partial \mathcal{B}_{0}^{\mathrm{N}} \quad \text { and } \quad \widehat{\operatorname{Div}} \widehat{\boldsymbol{P}}+\boldsymbol{t}_{0}^{\mathrm{p}}-\boldsymbol{P} \cdot \boldsymbol{N}=\mathbf{0} \quad \text { on } \mathcal{S}_{0}^{\mathrm{N}},
$$

in which $\boldsymbol{t}_{0}^{\mathrm{p}}$ denotes the prescribed traction on the Neumann portions of the boundaries $\partial \mathcal{B}_{0}$ and $\mathcal{S}_{0}$ denoted as $\partial \mathcal{B}_{0}^{\mathrm{N}}$ and $\mathcal{S}_{0}^{\mathrm{N}}$, respectively. The prescribed traction on the surfaces of the pores, i.e. $\boldsymbol{t}_{0}^{\mathrm{p}}$ on $\mathcal{S}_{0}^{\mathrm{N}}$, often vanishes unless in the case of pore pressure or similar effects. Balance of angular momentum results in the symmetry of Cauchy stresses in the bulk and on the surfaces equivalently expressed as

$$
\boldsymbol{P} \cdot \boldsymbol{F}^{\mathrm{t}}=\boldsymbol{F} \cdot \boldsymbol{P}^{\mathrm{t}} \quad \text { in } \mathcal{B}_{0} \quad \text { and } \quad \widehat{\boldsymbol{P}} \cdot \widehat{\boldsymbol{F}}^{\mathrm{t}}=\widehat{\boldsymbol{F}} \cdot \widehat{\boldsymbol{P}}^{\mathrm{t}} \quad \text { on } \mathcal{S}_{0},
$$

with $\boldsymbol{P}$ the bulk Piola stress and $\widehat{\boldsymbol{P}}$ the surface Piola stress. Note, in the absence of surfaces, i.e. $\widehat{\boldsymbol{P}}=\mathbf{0}$, the balance equations recover their conventional form for the bulk only problem. Remark: The boundary condition $(6)_{2}$ is essentially the balance of linear momentum on the surface $\mathcal{S}_{0}$ and is subject to the boundary condition $\widehat{\boldsymbol{P}} \cdot \widehat{\boldsymbol{N}}-\widetilde{\boldsymbol{t}}_{0}^{\mathrm{p}}=\mathbf{0}$ on $\mathcal{C}_{0}^{\mathrm{N}}$ where $\widetilde{\boldsymbol{t}}_{0}^{\mathrm{p}}$ denotes 
the prescribed traction on the Neumann portions of curve $\mathcal{C}_{0}^{\mathrm{N}}$. However, throughout this work we assume $\partial \mathcal{S}_{0}=\emptyset$ hence, $\mathcal{C}_{0}^{\mathrm{N}}=\emptyset$.

Finally, a Coleman-Noll procedure shows that the micro Piola stress in the bulk $\boldsymbol{P}$ and the micro Piola stress on the surface $\widehat{\boldsymbol{P}}$ for hyperelastic materials are given by

$$
\boldsymbol{P}=\frac{\partial \Psi}{\partial \boldsymbol{F}} \quad \text { and } \quad \widehat{\boldsymbol{P}}=\frac{\partial \widehat{\Psi}}{\partial \widehat{\boldsymbol{F}}} .
$$

Gurtin and Murdoch [10] assume that the surface Piola stress possesses the superficiality property $\widehat{\boldsymbol{P}} \cdot \boldsymbol{N}=\mathbf{0}$. Javili et al. [55] explain how this condition can be explained by rankdeficiency of $\widehat{\boldsymbol{F}}$ and eventually they prove that the superficiality of the surface stress is a consequence of a first-order bulk material, i.e. the stress is only a function of the deformation gradient.

\subsection{Connection between scales}

In this section macroscopic quantities are related to their micro counterparts through volume averaging over the RVE and fundamental reasoning. It proves convenient to define the following averaging operator in the material configuration $\langle\{\bullet\}\rangle_{\{\circ}$ as the integral of $\{\bullet\}$ over the domain $\{0\}$ divided by the volume $\mathscr{V}_{0}$ as

$$
\langle\{\bullet\}\rangle_{\{\circ\}}=\frac{1}{\mathscr{V}_{0}} \int_{\{\circ\}}\{\bullet\} \mathrm{d}\{0\},
$$

and in particular

$$
\langle\{\bullet\}\rangle_{\mathcal{B}_{0}}=\frac{1}{\mathscr{V}_{0}} \int_{\mathcal{B}_{0}}\{\bullet\} \mathrm{d} V \quad \text { and } \quad\langle\{\bullet\}\rangle_{\mathcal{S}_{0}}=\frac{1}{\mathscr{V}_{0}} \int_{\mathcal{S}_{0}}\{\bullet\} \mathrm{d} A
$$

The volume $\mathscr{V}_{0}$ is the total volume surrounded by the (external) boundary $\partial \mathcal{B}_{0}$ and can be understood as the total volume of the domains $\mathcal{B}_{0}$ and $\mathcal{V}_{0}$, that is

$$
\mathscr{V}_{0}=\int_{\mathcal{B}_{0}} \mathrm{~d} V+\int_{\mathcal{V}_{0}} \mathrm{~d} V
$$

where the first integral indicates the matrix volume and the second one the pore volume. The identity

$$
\int_{\partial \mathcal{B}_{0}} \boldsymbol{N} \otimes \boldsymbol{X} \mathrm{d} A=\mathscr{V}_{0} \boldsymbol{I}
$$

is proven in A.1. For a smoothly defined field $\{\bullet\}$ in both $\mathcal{B}_{0}$ and $\mathcal{V}_{0}$, we define the (classical) average operator as the average over the whole RVE without any subscript as

$$
\langle\{\bullet\}\rangle=\frac{1}{\mathscr{V}_{0}} \int_{\mathcal{B}_{0}}\{\bullet\} \mathrm{d} V+\frac{1}{\mathscr{V}_{0}} \int_{\mathcal{V}_{0}}\{\bullet\} \mathrm{d} V .
$$

If the field $\{\bullet\}$ is not smoothly defined within the whole domain, the average operator can be consistently redefined. In particular, for the Piola stress field $\boldsymbol{P}$ relation (13) reduces to

$$
\langle\boldsymbol{P}\rangle=\frac{1}{\mathscr{V}_{0}} \int_{\mathcal{B}_{0}} \boldsymbol{P} \mathrm{d} V+\frac{1}{\mathscr{V}_{0}} \int_{\mathcal{V}_{0}} \boldsymbol{P} \mathrm{d} V=\frac{1}{\mathscr{V}_{0}} \int_{\mathcal{B}_{0}} \boldsymbol{P} \mathrm{d} V=\langle\boldsymbol{P}\rangle_{\mathcal{B}_{0}},
$$


as the stress field vanishes within the pores. Also, since the deformation gradient cannot be defined within the pores, using the gradient theorem, the average operator (13) for $\boldsymbol{F}$ can be written as

$$
\langle\boldsymbol{F}\rangle=\frac{1}{\mathscr{V}_{0}} \int_{\mathcal{B}_{0}} \boldsymbol{F} \mathrm{d} V+\frac{1}{\mathscr{V}_{0}} \int_{\mathcal{S}_{0}} \widehat{\boldsymbol{\varphi}} \otimes \boldsymbol{N} \mathrm{d} A
$$

\subsubsection{Generalized average stress theorem}

In order to relate the macro stress to the microscopic one, motivated by classical homogenization, we develop an average stress theorem. However, the average stress theorem needs to be generalized in order to account for surface stresses. In this section, we propose a generalized average stress theorem and provide a proof. In the absence of surface stresses, this generalized format recovers the classical average stress theorem.

Theorem: Let $\boldsymbol{P}_{c}$ be a given constant stress tensor and $\partial \mathcal{B}_{0}$ be the external boundary of the domain $\mathcal{B}_{0}$ with outward normal $\boldsymbol{N}$ as shown in Fig. 2. If $\boldsymbol{P} \cdot \boldsymbol{N}=\boldsymbol{t}_{0}^{\mathrm{p}}=\boldsymbol{P}_{c} \cdot \boldsymbol{N}$ is prescribed on the entire $\partial \mathcal{B}_{0}$ and $\boldsymbol{t}_{0}^{\mathrm{p}}=\mathbf{0}$ holds on $\mathcal{S}_{0}$, then $\langle\boldsymbol{P}\rangle_{\mathcal{B}_{0}}+\langle\widehat{\boldsymbol{P}}\rangle_{\mathcal{S}_{0}}=\boldsymbol{P}_{c}$.

Pro of. In order to prove the generalized average stress theorem, we employ the identity

$$
\langle\boldsymbol{P}\rangle=\frac{1}{\mathscr{V}_{0}} \int_{\partial \mathcal{B}_{0}} \boldsymbol{t}_{0}^{\mathrm{p}} \otimes \boldsymbol{X} \mathrm{d} A+\frac{1}{\mathscr{V}_{0}} \int_{\mathcal{S}_{0}} \boldsymbol{t}_{0}^{\mathrm{p}} \otimes \widehat{\boldsymbol{X}} \mathrm{d} A-\frac{1}{\mathscr{V}_{0}} \int_{\mathcal{S}_{0}} \widehat{\boldsymbol{P}} \mathrm{d} A
$$

which is proven in A.2. The second integral vanishes since the prescribed traction is zero on $\mathcal{S}_{0}$. Also, $\langle\boldsymbol{P}\rangle_{\mathcal{B}_{0}}=\langle\boldsymbol{P}\rangle$ according to Eq. (14) as the pores are stress-free. Therefore, we have:

$$
\begin{aligned}
\langle\boldsymbol{P}\rangle_{\mathcal{B}_{0}}+\langle\widehat{\boldsymbol{P}}\rangle_{\mathcal{S}_{0}} & =\frac{1}{\mathscr{V}_{0}} \int_{\partial \mathcal{B}_{0}} \boldsymbol{t}_{0}^{\mathrm{p}} \otimes \boldsymbol{X} \mathrm{d} A \\
& =\frac{1}{\mathscr{V}_{0}} \int_{\partial \mathcal{B}_{0}} \boldsymbol{P}_{\mathrm{c}} \cdot \boldsymbol{N} \otimes \boldsymbol{X} \mathrm{d} A=\frac{1}{\mathscr{V}_{0}} \boldsymbol{P}_{\mathrm{c}} \cdot \int_{\partial \mathcal{B}_{0}} \boldsymbol{N} \otimes \boldsymbol{X} \mathrm{d} A .
\end{aligned}
$$

Using the identity (12), relation (17) simplifies to

$$
\langle\boldsymbol{P}\rangle_{\mathcal{B}_{0}}+\langle\widehat{\boldsymbol{P}}\rangle_{\mathcal{S}_{0}}=\frac{1}{\mathscr{V}_{0}} \boldsymbol{P}_{\mathrm{c}} \cdot\left[\mathscr{V}_{0} \boldsymbol{I}\right]=\boldsymbol{P}_{\mathrm{c}}
$$

The average stress theorem tells us when a body is subject to traction boundary conditions as defined above, the stresses averaged over the entire body plus the surface stresses averaged over the entire surfaces of the pores is the same as $\boldsymbol{P}_{\mathrm{c}}$ regardless the complexity of the stress field within the RVE domain. In view of the micro-to-macro transition, the average stress theorem motivates the macro stress to be defined by sum of the average bulk and surface stresses as

$$
\begin{aligned}
{ }^{\mathrm{M}} \boldsymbol{P} & :=\langle\boldsymbol{P}\rangle+\langle\widehat{\boldsymbol{P}}\rangle_{\mathcal{S}_{0}}=\langle\boldsymbol{P}\rangle_{\mathcal{B}_{0}}+\langle\widehat{\boldsymbol{P}}\rangle_{\mathcal{S}_{0}} \\
& =\frac{1}{\mathscr{V}_{0}} \int_{\mathcal{B}_{0}} \boldsymbol{P} \mathrm{d} V+\frac{1}{\mathscr{V}_{0}} \int_{\mathcal{S}_{0}} \widehat{\boldsymbol{P}} \mathrm{d} A=\frac{1}{\mathscr{V}_{0}} \int_{\partial \mathcal{B}_{0}} \boldsymbol{t}_{0}^{\mathrm{p}} \otimes \boldsymbol{X} \mathrm{d} A .
\end{aligned}
$$


Clearly, if $\widehat{\boldsymbol{P}}=\mathbf{0}$, the relation (18) reduces to the classical average stress theorem. More interestingly, the definition of the macro stress as the surface integral

$$
{ }^{\mathrm{M}} \boldsymbol{P}:=\frac{1}{\mathscr{V}_{0}} \int_{\partial \mathcal{B}_{0}} \boldsymbol{t}_{0}^{\mathrm{p}} \otimes \boldsymbol{X} \mathrm{d} A,
$$

is identical to its format in classical homogenization.

\subsubsection{Generalized average deformation gradient theorem}

In order to relate the macro deformation gradient to the micro deformation gradient, motivated by the classical homogenization, we develop an average deformation gradient theorem. Note, the term average strain theorem is frequently used due to the origins of this theory in linear elasticity. In principle, the average deformation gradient theorem needs to be generalized in order to account for the surface deformation gradients. However, it turns out that, in contrast to the average stress theorem, the generalized format of the deformation gradient theorem remains identical to the classical one. This can be explained by the fact that the classical theorem neglects only the surface stresses but the surface deformations are intrinsically accounted for.

Theorem: Let $\boldsymbol{F}_{c}$ be a given constant deformation gradient tensor and $\partial \mathcal{B}_{0}$ be the external boundary of the domain $\mathcal{B}_{0}$ with outward normal $\boldsymbol{N}$ as shown in Fig. 2. If $\boldsymbol{\varphi}=\boldsymbol{F}_{c} \cdot \boldsymbol{X}$ is prescribed on the entire $\partial \mathcal{B}_{0}$, then $\langle\boldsymbol{F}\rangle=\boldsymbol{F}_{c}$.

Pro of. In order to prove the generalized average deformation gradient theorem, we employ the gradient theorem taking the orientations of surface normals carefully into account as

$$
\begin{aligned}
\langle\boldsymbol{F}\rangle & =\frac{1}{\mathscr{V}_{0}} \int_{\mathcal{B}_{0}} \boldsymbol{F} \mathrm{d} V+\frac{1}{\mathscr{V}_{0}} \int_{\mathcal{S}_{0}} \widehat{\boldsymbol{\varphi}} \otimes \boldsymbol{N} \mathrm{d} A \\
& =\frac{1}{\mathscr{V}_{0}} \int_{\partial \mathcal{B}_{0}} \boldsymbol{\varphi} \otimes \boldsymbol{N} \mathrm{d} A+\underbrace{\frac{1}{\mathscr{V}_{0}} \int_{\mathcal{S}_{0}} \widehat{\varphi} \otimes \boldsymbol{N} \mathrm{d} A-\frac{1}{\mathscr{V}_{0}} \int_{\mathcal{S}_{0}} \widehat{\varphi} \otimes N \mathrm{~d} A}_{=0} \\
& =\frac{1}{\mathscr{V}_{0}} \int_{\partial \mathcal{B}_{0}} \boldsymbol{\varphi} \otimes \boldsymbol{N} \mathrm{d} A .
\end{aligned}
$$

Next, we impose $\varphi=\boldsymbol{F}_{\mathrm{c}} \cdot \boldsymbol{X}$, use the identity (12) and the symmetry property of the identity tensor as

$$
\begin{aligned}
\langle\boldsymbol{F}\rangle & =\frac{1}{\mathscr{V}_{0}} \int_{\partial \mathcal{B}_{0}} \boldsymbol{\varphi} \otimes \boldsymbol{N} \mathrm{d} A=\frac{1}{\mathscr{V}_{0}} \int_{\partial \mathcal{B}_{0}} \boldsymbol{F}_{\mathrm{c}} \cdot \boldsymbol{X} \otimes \boldsymbol{N} \mathrm{d} A \\
& =\frac{1}{\mathscr{V}_{0}} \boldsymbol{F}_{\mathrm{c}} \cdot \int_{\partial \mathcal{B}_{0}} \boldsymbol{X} \otimes \boldsymbol{N} \mathrm{d} A=\frac{1}{\mathscr{V}_{0}} \boldsymbol{F}_{\mathrm{c}} \cdot\left[\mathscr{V}_{0} \boldsymbol{I}^{\mathrm{t}}\right]=\boldsymbol{F}_{\mathrm{c}} .
\end{aligned}
$$

The average deformation gradient theorem tells us that when a body is subject to the linear displacement boundary conditions defined above, with $\boldsymbol{F}_{\mathrm{c}}$ being a constant tensor, the deformation gradient averaged over the entire body is the same as $\boldsymbol{F}_{\mathrm{c}}$ regardless the complexity of 
the deformation within the RVE domain. In view of the micro-to-macro transition, the average deformation gradient theorem motivates the definition of the macro deformation gradient as

$$
{ }^{\mathrm{M}} \boldsymbol{F}:=\langle\boldsymbol{F}\rangle=\frac{1}{\mathscr{V}_{0}} \int_{\mathcal{B}_{0}} \boldsymbol{F} \mathrm{d} V+\frac{1}{\mathscr{V}_{0}} \int_{\mathcal{S}_{0}} \widehat{\boldsymbol{\varphi}} \otimes \boldsymbol{N} \mathrm{d} A=\frac{1}{\mathscr{V}_{0}} \int_{\partial \mathcal{B}_{0}} \boldsymbol{\varphi} \otimes \boldsymbol{N} \mathrm{d} A
$$

\subsubsection{Generalized Hill-Mandel condition}

Motivated by the generalized stress and deformation gradient theorems, the macro stress ${ }^{\mathrm{M}} \boldsymbol{P}$ and the macro deformation gradient ${ }^{\mathrm{M}} \boldsymbol{F}$ are defined by relations (18) and (20), respectively. Next, we must impose an incremental energy equivalence between the macro and micro scales in a generalized fashion to account for the surface stresses of the pores. The incremental energy equivalence between the scales is known as Hill-Mandel condition. We propose the following generalized Hill-Mandel condition

$$
\langle\boldsymbol{P}: \delta \boldsymbol{F}\rangle+\langle\widehat{\boldsymbol{P}}: \delta \widehat{\boldsymbol{F}}\rangle_{\mathcal{S}_{0}}-{ }^{\mathrm{M}} \boldsymbol{P}: \delta^{\mathrm{M}} \boldsymbol{F} \stackrel{!}{=} 0
$$

The next task is to seek suitable boundary conditions on the RVE which satisfy the generalized Hill-Mandel condition (21). In order to do so, we introduce the generalized Hill's identity, proven in A.3, which states that

$$
\begin{aligned}
\langle\boldsymbol{P}: \delta \boldsymbol{F}\rangle+\langle\widehat{\boldsymbol{P}}: \delta \widehat{\boldsymbol{F}}\rangle_{\mathcal{S}_{0}} & -{ }^{\mathrm{M}} \boldsymbol{P}: \delta^{\mathrm{M}} \boldsymbol{F} \\
& =\frac{1}{\mathscr{V}_{0}} \int_{\partial \mathcal{B}_{0}}\left[\delta \boldsymbol{\varphi}-\delta^{\mathrm{M}} \boldsymbol{F} \cdot \boldsymbol{X}\right] \cdot\left[\boldsymbol{t}_{0}^{\mathrm{p}}-{ }^{\mathrm{M}} \boldsymbol{P} \cdot \boldsymbol{N}\right] \mathrm{d} A .
\end{aligned}
$$

The generalized Hill's identity (22) essentially expresses the left-hand side of the condition (21) in terms of a surface integral over the external boundary of the RVE $\partial \mathcal{B}_{0}$. This has the advantage that the generalized Hill-Mandel condition, which is essentially a volume integral, can be transformed to a boundary integral and eventually identifies the appropriate boundary conditions that guarantee the incremental energy equivalence between the scales. In order to satisfy the generalized Hill-Mandel condition, the right-hand side of Eq. (22) should identically vanish. Here, we list several conditions, deduced from the right-hand side of Eq. (22), that sufficiently satisfy the generalized Hill-Mandel condition. 


$$
\begin{array}{ll}
\text { Voigt } \rightsquigarrow \varphi={ }^{\mathrm{M}} \boldsymbol{F} \cdot \boldsymbol{X} & \text { in } \mathcal{B}_{0},
\end{array}
$$

linear displacement (Taylor) assumption $\Rightarrow$ Voigt bound,

$$
\mathrm{DBC} \rightsquigarrow \varphi={ }^{\mathrm{M}} \boldsymbol{F} \cdot \boldsymbol{X} \quad \text { on } \partial \mathcal{B}_{0},
$$

linear displacement boundary condition (DBC),

$$
\mathrm{PBC} \rightsquigarrow\left[\boldsymbol{\varphi}-{ }^{\mathrm{M}} \boldsymbol{F} \cdot \boldsymbol{X}\right]: \text { periodic },\left[\boldsymbol{t}_{0}^{\mathrm{p}}-{ }^{\mathrm{M}} \boldsymbol{P} \cdot \boldsymbol{N}\right]: \text { anti-periodic on } \partial \mathcal{B}_{0},
$$

periodic displacement and anti-periodic traction boundary condition,

$$
\text { ТВC } \rightsquigarrow \boldsymbol{t}_{0}^{\mathrm{p}}={ }^{\mathrm{M}} \boldsymbol{P} \cdot \boldsymbol{N} \quad \text { on } \partial \mathcal{B}_{0},
$$

constant traction boundary condition (TBC),

$$
\begin{aligned}
\text { Reuss } & \rightsquigarrow \boldsymbol{t}_{0}^{\mathrm{p}}={ }^{\mathrm{M}} \boldsymbol{P} \cdot \boldsymbol{N} \\
& \text { constant traction (Sachs) assumption } \Rightarrow \text { Reuss bound . }
\end{aligned}
$$$$
\text { in } \mathcal{B}_{0} \text {, }
$$

Note, for the periodic boundary conditions (PBC), anti-periodic traction $t_{0}^{\mathrm{p}}$ satisfies the antiperiodicity of $\left[\boldsymbol{t}_{0}^{\mathrm{p}}-{ }^{\mathrm{M}} \boldsymbol{P} \cdot \boldsymbol{N}\right]$ since ${ }^{\mathrm{M}} \boldsymbol{P} \cdot \boldsymbol{N}$ is anti-periodic itself due to anti-periodicity of the boundary normals. For classical homogenization without energetic surfaces at the microscale, it is well-known that the Reuss condition results in the "most compliant" and the Voigt condition results in the "most stiff" response. In the remainder of this manuscript we limit ourselves to the PBC in the numerical examples.

Remark: It can be shown that using the definitions of the macro stress $(18)_{2}$ and macro deformation gradient (20), all the aforementioned boundary conditions satisfy the balance of angular momentum on the macroscale. The proof is straightforward and follows the same steps as for classical homogenization [56].

\subsection{Constitutive laws for the microscopic bulk and surface}

In order to compute the microscopic response, constitutive laws on the RVE need to be specified for both the bulk and the surfaces of the pores. For the bulk at the microscale we consider a hyperelastic neo-Hookean constitutive model with the free energy density

$$
\Psi(\boldsymbol{F})=\frac{1}{2} \lambda \log ^{2} J+\frac{1}{2} \mu[\boldsymbol{F}: \boldsymbol{F}-3-2 \log J],
$$

where $\lambda$ and $\mu$ are the Lamé parameters and $J:=\operatorname{Det} \boldsymbol{F}>0$ is the Jacobian determinant. Motivated by the material response of the bulk, we consider a neo-Hookean model for the surface free energy density

$$
\widehat{\Psi}(\widehat{\boldsymbol{F}})=\frac{1}{2} \widehat{\lambda} \log ^{2} \widehat{J}+\frac{1}{2} \widehat{\mu}[\widehat{\boldsymbol{F}}: \widehat{\boldsymbol{F}}-2-2 \log \widehat{J}],
$$


where $\widehat{\lambda}$ and $\widehat{\mu}$ are the surface Lamé parameters and $\widehat{J}:=\widehat{\operatorname{Det}} \widehat{\boldsymbol{F}}>0$ is the surface Jacobian determinant [57]. We note that in the surface energy surface tension is neglected as, in solids, it is generally small compared to elastic resistance of the surface.

Remark: We assume positive values for the surface elastic parameters in this work. It has been observed experimentally and also in atomistic simulations $[58,59]$ that surface material parameters could assume negative values. The admissible range for surface material parameters have been studied in [60]. Having negative surface elastic parameters, does not change the methodology or the conclusion of this work. The same framework can be employed by replacing the surface parameters with the negative ones and also, the size effect will be observed. However, negative surface parameters lead to a "smaller is weaker"response in contrast to the more celebrated "smaller is stronger"response. From a mathematical point of view, positive surface parameters guarantee the pointwise stability and ellipticity regardless of the pore size. That is not the case for negative surface parameters as the overall response may show instabilities for very small pores with negative surface parameters. Also, negative surface parameters induce a sensitivity with respect to the mesh size of the finite element method. The response of nano-porous materials with negative surface parameters shall be further investigated in a subsequent contribution.

\section{Examples}

The objective of this section is to elucidate the theory via a series of numerical examples. For simplicity, we limit this study to two-dimensional examples keeping in mind that the developed theory is based on a fully three-dimensional setting. Details of the finite element implementation of surfaces for two-dimensional and three-dimensional problems are given in [22] and [23], respectively. Furthermore, we present an analytical estimate for two-dimensional linear elasticity and compare it with the numerical results. For the microscopic bulk response, the material parameters are set to $\lambda=12.1667$ and $\mu=8$. The radius of the pore is denoted $r$ and the volume fraction $f$. The surface material property is chosen as $\widehat{\mu} / \mu=1 \mathrm{e}-6$. Since for the two-dimensional theory the surface simplifies to a line, one surface material parameter suffices to describe the elastic response of the surface and therefore, $\widehat{\lambda}=0$ is assumed.

\subsection{Analytical approach}

The analytical solution for a material which has cylindrical nano voids with energetic surfaces has been studied by Duan et al. [51] under small strain and linear elasticity assumptions. For an analytical homogenization framework that accounts for size effects with applications to multilayered orthotropic composite, see [61]. In [51] the authors compute four effective properties (in-plane bulk modulus, axial shear modulus, axial Young's modulus and axial Poisson's ratio) using an extension of the composite cylinders method [62] and for the fifth (in-plane shear modulus) they utilize the self-consistent composite cylinders method [63]. Using the formulas and methodology of Duan et al. [51] we obtain the effective in-plane bulk 
and shear modulus as

$$
\begin{aligned}
\kappa^{\mathrm{eff}} & =\frac{[\lambda+\mu+f \mu][\widehat{\mu} / r]+[1-f][1-\widehat{\nu}][\lambda+\mu] \mu}{[1-f][\widehat{\mu} / r]+[1-\widehat{\nu}][\mu+f \lambda+f \mu]}, \\
\mu^{\mathrm{eff}} & =\mu[1-f]^{3}[\lambda+\mu] \frac{[2 \lambda+3 \mu+2 f \lambda+5 f \mu][\widehat{\mu} / r]+[1-f][1-\widehat{\nu}][\lambda+\mu] \mu}{\alpha[\widehat{\mu} / r]+[1-f][\lambda+\mu][1-\widehat{\nu}] \beta \mu+[\lambda+2 \mu] \sqrt{\gamma}},
\end{aligned}
$$

respectively, where

$$
\begin{aligned}
\alpha & :=2 f[\lambda+\mu]\left[2 f^{2} \lambda+6 f^{2} \mu-\lambda-3 f \mu\right]-\mu[2 \lambda+3 \mu]+f^{4} \mu[2 \lambda+5 \mu], \\
\beta & :=\left[4 f^{2}-2 f\right] \lambda+\left[f^{3}+5 f^{2}-f-1\right] \mu, \\
\gamma:= & {\left[\left[2 \lambda+3 \mu+2 f^{4} \lambda+5 f^{4} \mu\right][\widehat{\mu} / r]+\left[1-f^{4}\right][1-\widehat{\nu}][\lambda+\mu] \mu\right]^{2} } \\
& -12 f^{2}[[\lambda+\mu+f \mu][\widehat{\mu} / r]+[1-f][1-\widehat{\nu}][\lambda+\mu] \mu]^{2},
\end{aligned}
$$

with $\widehat{\nu}=\widehat{\lambda} /[\widehat{\lambda}+2 \widehat{\mu}]$ being the surface Poisson's ratio [23].

Based on the analytical solution we estimate the response of the microstructure at two extremes of (i) no surface effect and (ii) strong surface effect.

(i) For extremely low surface to volume ratio $(r \rightarrow \infty)$, the effective response tends asymptotically to

$$
\begin{aligned}
& \kappa^{\mathrm{eff}}=\frac{\mu[1-f][\lambda+\mu]}{\mu+f \lambda+f \mu}, \\
& \mu^{\mathrm{eff}}=\frac{\mu[1-f]^{3}[\lambda+\mu]}{\beta+[\lambda+2 \mu] \sqrt{\left[f^{3}+f^{2}+f+1\right]^{2}-12 f^{2}}} .
\end{aligned}
$$

(ii) For extremely high surface to volume ratio $(r \rightarrow 0)$, the effective response tends asymptotically to

$$
\begin{aligned}
& \kappa^{\mathrm{eff}}=\frac{\lambda+\mu+f \mu}{1-f}, \\
& \mu^{\mathrm{eff}}=\frac{\mu[1-f]^{3}[\lambda+\mu][2 \lambda+3 \mu+2 f \lambda+5 f \mu]}{\alpha+[\lambda+2 \mu] \sqrt{\left[2 \lambda+3 \mu+2 f^{4} \lambda 5 f^{4} \mu\right]^{2}-12 f^{2}[\lambda+\mu+f \mu]^{2}}} .
\end{aligned}
$$

Note that the no-surface-effect limit furnishes the classical solution and we expect intuitively the effective behavior to be independent of the surface elastic parameters. The analytical solution agrees with this intuition. For the strong-surface-effect limit, we expect that the surface parameters determine the material response. However, the solution is rather counter-intuitive as for extremely strong surface effect, the overall behavior of the material is independent of the surface parameters. This finding can be explained by the fact that extremely strong surfaces "roughly speaking" function similar to rigid inclusions and therefore the overall response does not depend on the surface. Surprisingly, this implies that there is a limit to the response of nano-porous materials and to the notion of "smaller is stronger". This conclusion is extremely important when fabricating nano-porous materials since it could suggest an optimal size for the pores where the strongest response for the lowest fabrication cost is achieved. 


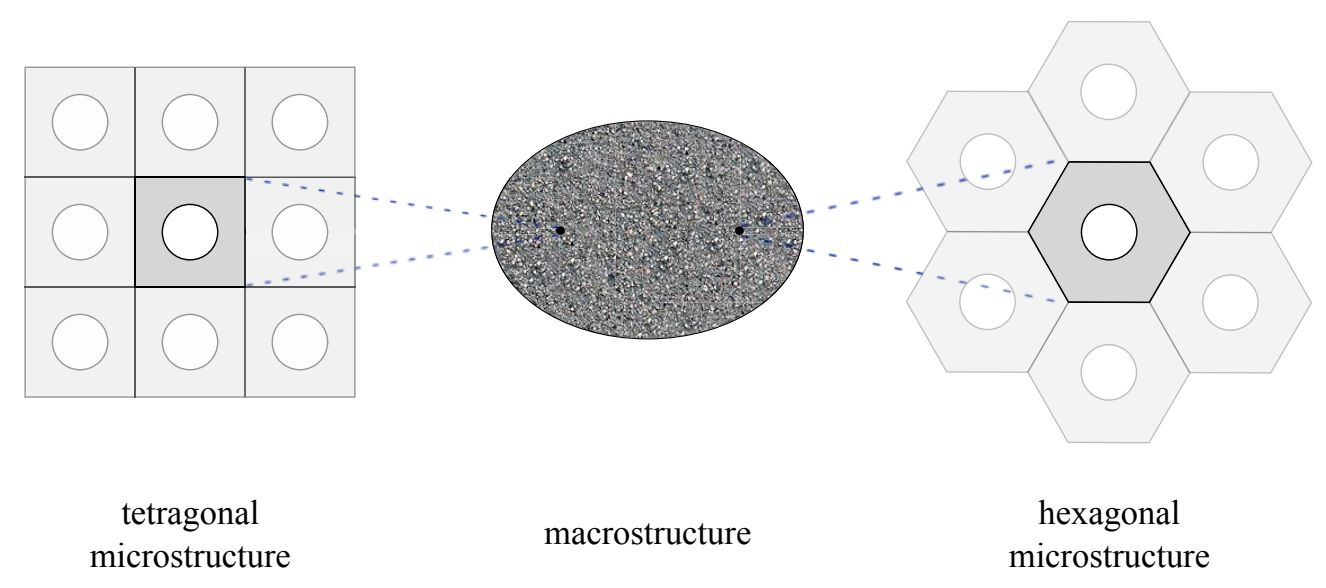

Fig. 3 Illustration of tetragonal and hexagonal microstructures.

\subsection{Numerical approach}

In this section we run several numerical examples to illustrate the influence of surfaces on the overall material response. All studies are performed for two different microstructures with tetragonal and hexagonal RVE arrangements, shown in Fig. 3. We investigate two different load cases of simple extension and simple shear with the associated macro deformation gradient applied on the RVE

$$
{ }^{\mathrm{M}} \boldsymbol{F}=\left[\begin{array}{cc}
1+\xi & 0 \\
0 & 1
\end{array}\right] \quad \text { and } \quad{ }^{\mathrm{M}} \boldsymbol{F}=\left[\begin{array}{cc}
1 & \xi \\
0 & 1
\end{array}\right]
$$

where $\xi$ indicates the amount of deformation.

Remark: The motivation to study two different microstructures is that the analytical approximation is based on concentric cylinders which suggests a better agreement for hexagonal microstructure compared to the tetragonal one. The results show that this is correct only for the load case of simple extension.

\subsubsection{Comparison between analytical and numerical approaches}

The first example compares the overall response from the analytical solution to the numerical one for both tetragonal and hexagonal microstructures. Figure 4 gathers all the results for different volume fractions. The graphs show a macroscopic apparent property versus pore radius. The apparent property of interest here is the macro Piola stress in $x x$-direction and $x y$-direction for the extension and shear load cases, respectively. The results correspond to $\xi=2 \%$ with macro deformation gradients (31). The numerical results are computed based on the linearized elasticity model associated with the material response (23) and (24). The linearized material response for the bulk associated with the free energy (23) is standard and leads to the isotropic Hookean material model of linear elasticity theory. However, this is not the case for the surface. Linearization of the stress associated with the free surface energy (23) is non-standard and care must be taken when linearizing. In particular, the surface linear strain 

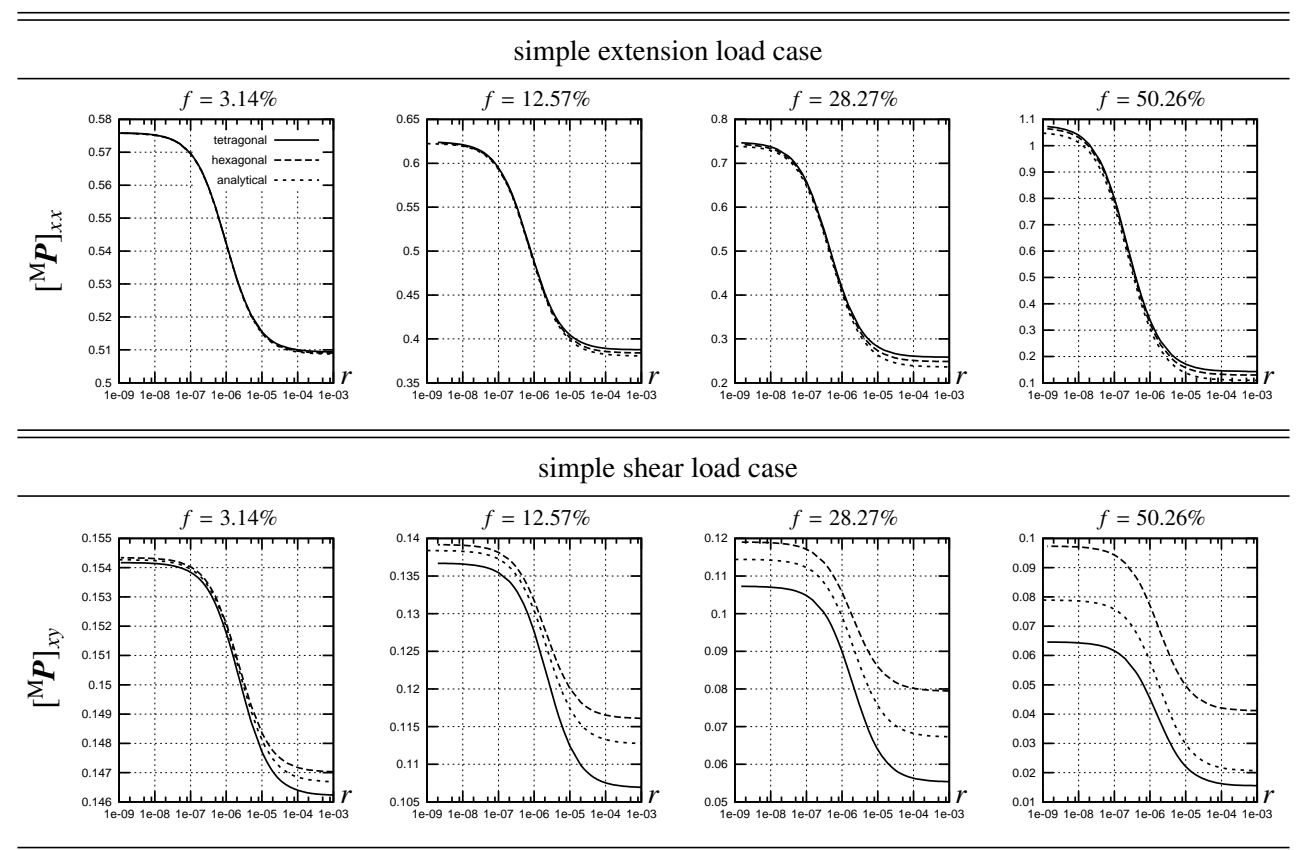

Fig. 4 Comparison between analytical and (linear) numerical solutions for overall response of the microstructure in two different cases of simple extension and simple shear at $2 \%$ deformation. The graphs show the overall Piola stress ( $x x$-component and $x y$-component in extension and shear case, respectively) versus pore radius of the RVE. Numerical results are obtained for both tetragonal and hexagonal microstructures and for different pore to volume ratios $f$.

tensor is not the symmetric part of the surface displacement gradient. For further details and derivations, see [24].

Figure 4 clearly identifies the bounds to the material response for all the load-cases and volume fractions. The lower bound corresponds to the classical solution where the surface elasticity of the pores is neglected. The classical solution (lower bound) is only a function of the volume fraction and properties of the matrix material. Taking surface elasticity into account, we observe a stronger material response for smaller dimensions which proves the ability of the proposed methodology to capture the size effect. At very small dimensions, the material response is strongly influenced by surfaces. In the limit of an extremely strong surface effect, the pores behave similar to rigid inclusions. This leads to the saturation of the overall behavior and defines an upper limit to the material response as a function of volume fraction and matrix properties.

Further analysis of the results in Fig. 4 show that for the simple extension load case, the analytical solution underestimates both numerical solutions associated with the tetragonal and hexagonal arrangements but, overall it closely estimates the numerical solution. In the case of simple shear, the analyical solution lies between the two different microstructures and for lower volume fractions it is closer to the hexagonal microstructure while it better estimates the tetragonal microstructure for higher volume fractions. Overall, in the case of simple shear, a 
very distinct difference in the response is observed between the two different microstructures and the analytical solution is far less accurate compared to the simple extension.

\subsubsection{Stress distribution for various sizes and volume fractions}

Figures 5-6 show the stress distribution within the RVE for the tetragonal microstructure for the simple extension and simple shear load cases, respectively. The numerical simulations are performed for different volume fractions and also for various sizes of the RVE. For all the examples, the results correspond to $\xi=5 \%$ with the macro deformation gradients given in (31). Analogous studies have been carried out on hexagonal microstructure in Figs. 7-8. The first column in Figs. 5-8 correspond to a pore radius of $r=1 \mathrm{e}-4$ indicating a relatively large RVE and thus a relatively small surface effect. The results for $r=1 \mathrm{e}-4$ resemble the classical solution with no surface effect. The pore radius for the results in the second column is chosen as $r=1 \mathrm{e}-6$ resulting in a moderate influence of the surface on the overall response of the material. A very strong surface response is illustrated in the last column with $r=1 \mathrm{e}-8$.

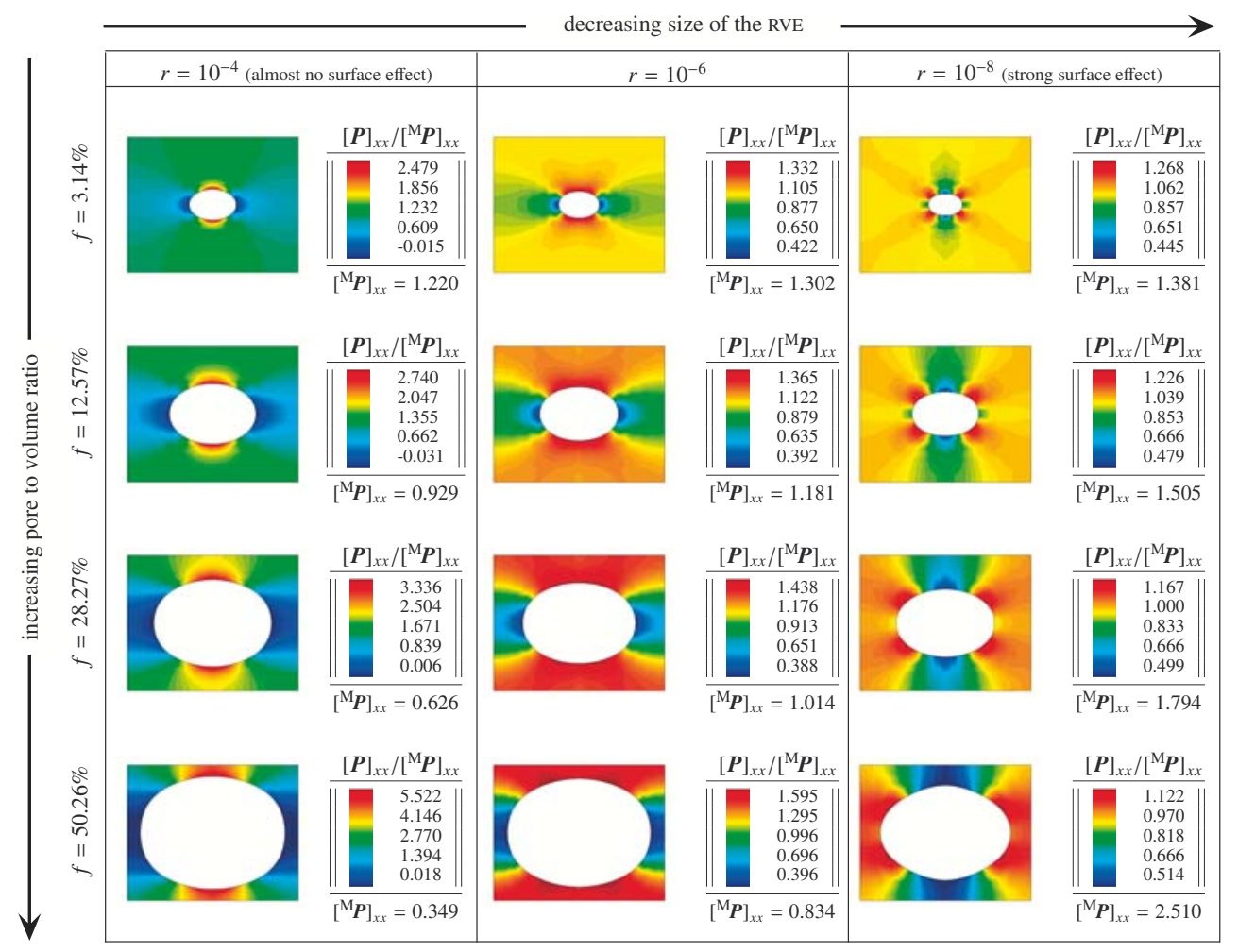

Fig. 5 (online colour at: www.gamm-mitteilungen.org) Stress distribution of a tetragonal microstructure under simple extension for various sizes of RVE and pore-to-volume ratio.

The stress distribution in Figs. 5-8 is normalized by the corresponding component of the resultant macro stress for better clarity. Overall, we observe the same trend for tetragonal and hexagonal microstructures. For a given volume fraction and size, the stress distributions as 


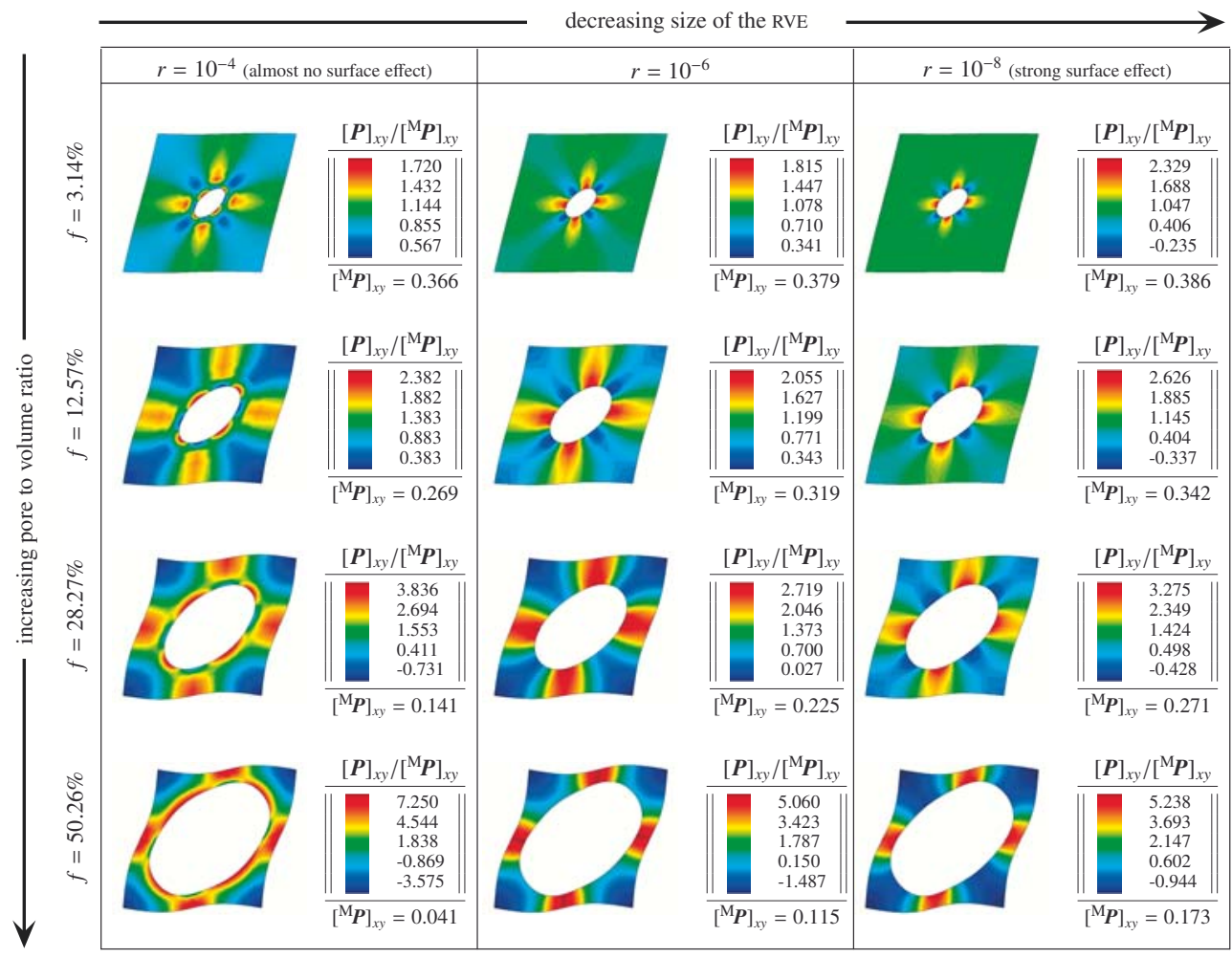

Fig. 6 (online colour at: www.gamm-mitteilungen.org) Stress distribution of a tetragonal microstructure under simple shear for various sizes of RVE and pore-to-volume ratio.

well as the macro stresses are roughly the same for both microstructures. The two microstructures show more similarities in the case of simple extension load case compared to the simple shear load case though. Figures 5-8 contain a lot of information and several conclusions can be drawn. In the following, we address a few important aspects.

Consider the first column of the results shown in Fig. 5 and Fig. 7 in the case of simple extension. Increasing the volume fraction decreases the overall response, here the macro stress in the $x x$-direction. This result is rather intuitive since larger volume fractions correspond to less solid material and hence, less resistance. Note, the macro stress for $f=50.26 \%$ is $70 \%$ smaller than the one for $f=3.14 \%$. The second column of Figs. 5 and 7 show a similar trend. We observe the overall response becomes softer by increasing the volume fraction $f$. However, the macro stress for $f=50.26 \%$ is only about $40 \%$ less than the one for $f=3.14 \%$. That can be explained by the fact that increasing the volume fraction reduces the bulk material but increases the surface material. Since the middle column has moderate surface effects, the overall response has decreased, but less than the first column. In the third column a very strong surface effect is observed as the pores are extremely small. Increasing the volume fraction, reduces the bulk material but increases the surface material. In fact, the surface effect is so strong that increasing the volume fraction stiffens the overall material behavior. The macro stress for $f=50.26 \%$ is about $100 \%$ more than the one for $f=3.14 \%$. These results clearly 


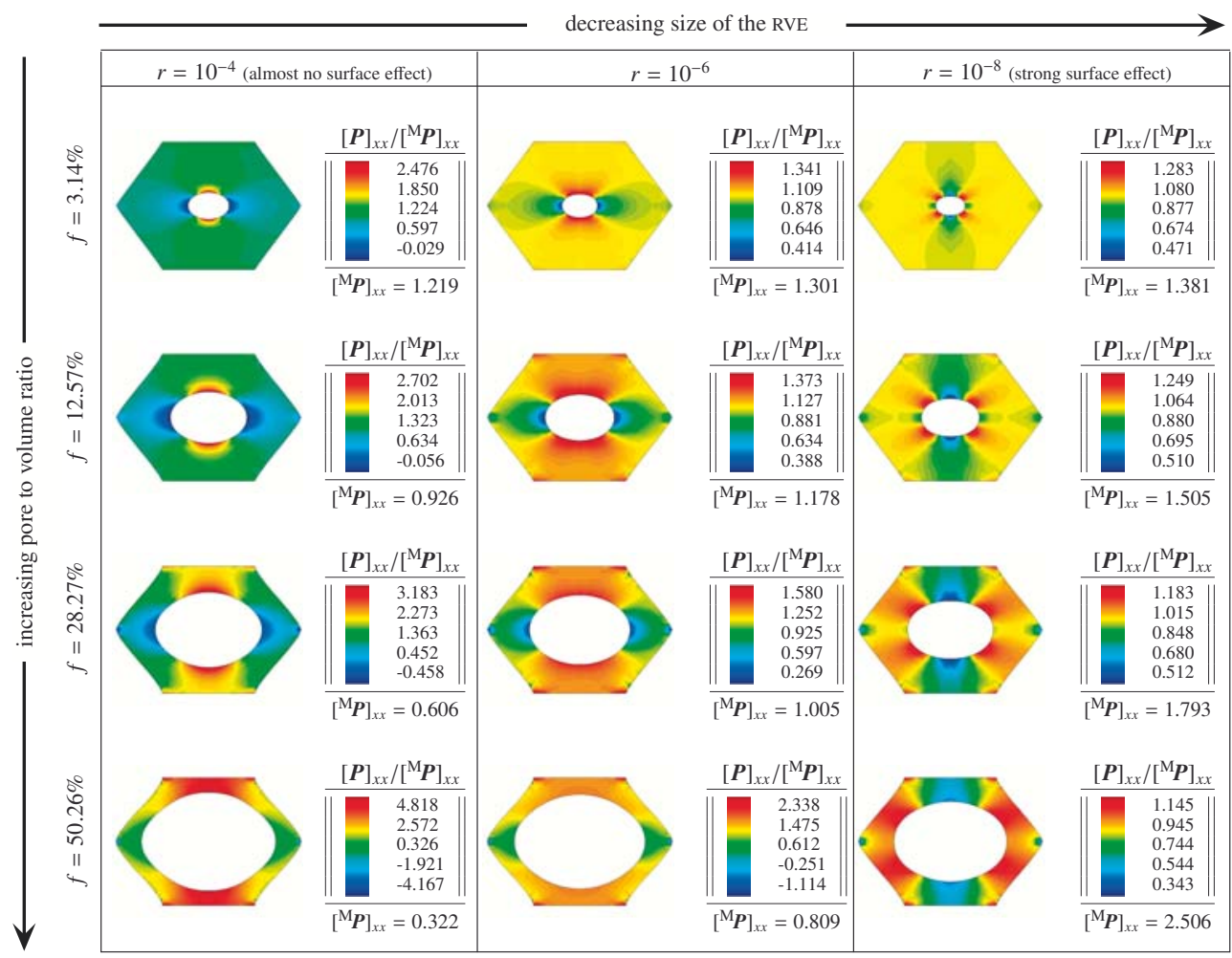

Fig. 7 (online colour at: www.gamm-mitteilungen.org) Stress distribution of a hexagonal microstructure under simple extension for various sizes of RVE and pore-to-volume ratio.

show that in the third column the overall response is dominated by the surfaces of the pores and indicates the importance of surfaces when the pore size is very small. It also explains why nano-porous materials are very good candidates for light-weight structures.

We briefly perform the same analysis for the simple shear load case, i.e. Figs. 6 and 8. As for the simple extension case, we observe a softer material response for higher volume fractions in the absence of surface effect, i.e. first column. In the second column, i.e. a moderate surface effect, the macro stress is decreased less compared to the first column. Interestingly, and in contrast to the simple extension load case, even in the third column we observe an overall softening response by increasing the volume fraction. This can be justified by the fact that the surface elastic response resist the area change and in these two-dimensional examples tends to retain the original perimeter of the pore. In the case of simple extension, we observe a very strong surface effect as applying an extension to the bulk increases the length (perimeter) of the pore. However, prescribing simple shear on the RVE deforms the original configuration roughly in a volume-preserving manner and therefore, the perimeter of the pores change less leading to a weaker surface influence on the overall response.

Next, we study the overall response for a fixed volume fraction $f$, i.e. we consider rows instead of columns in Figs. 5-8. For both microstructures and for both load cases, decreasing the size of the RVE results in increasing the overall response for all volume fractions. That is, 


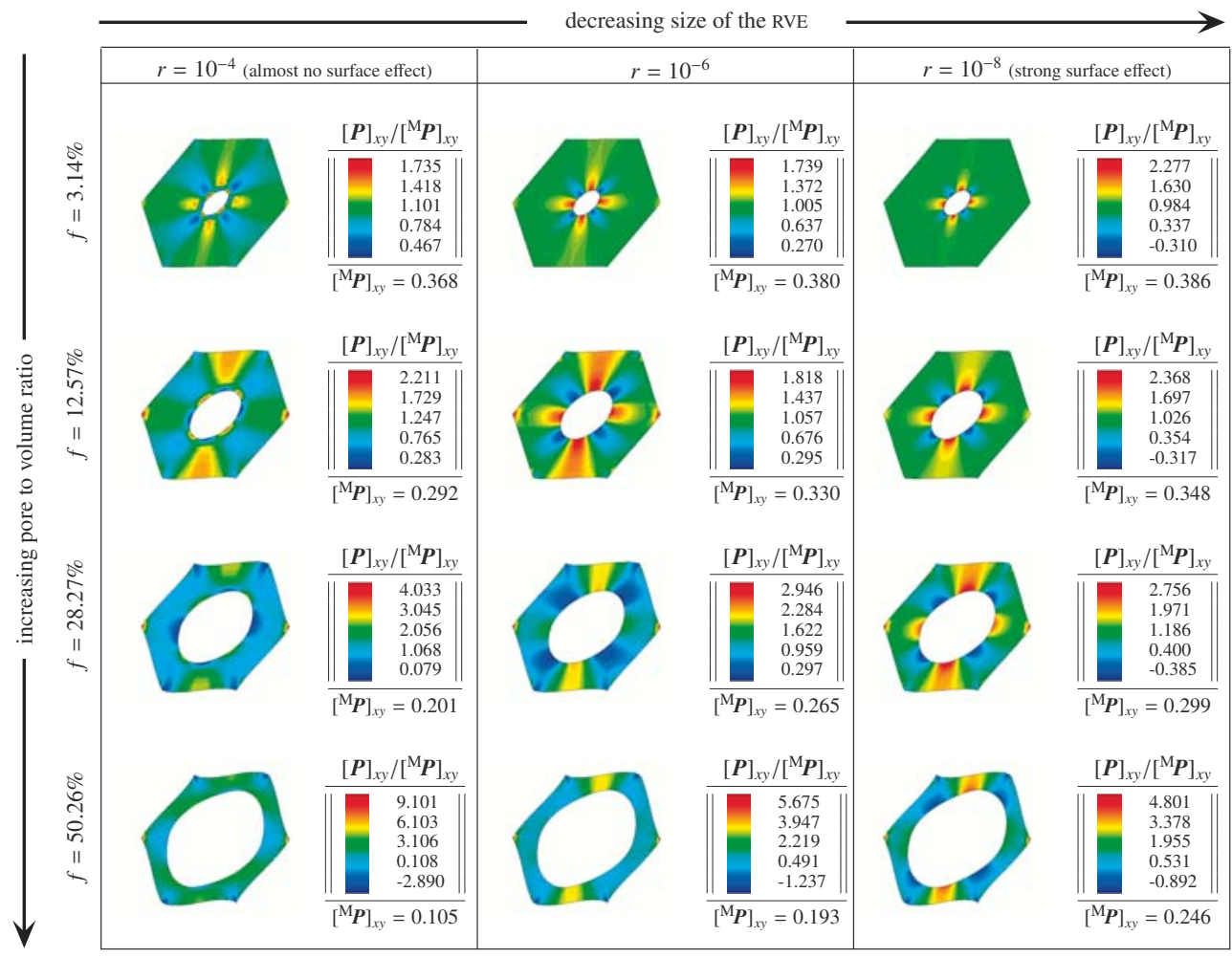

Fig. 8 (online colour at: www.gamm-mitteilungen.org) Stress distribution of a hexagonal microstructure under simple shear for various sizes of RVE and pore-to-volume ratio.

for a given row in Figs. 5-8, we observe a successive increase of the overall response when moving from the first column to the third column. This can be interpreted by the fact that decreasing the size, increases the surface influence. The overall response of the third column is increased more compared to the first column for higher volume fractions. Also, similar to the previous discussion, the simple extension load case shows a more dominant surface effect compared to the simple shear load case. In particular, for the simple extension and at volume fraction of $f=50.26 \%$ the overall response of the third column is almost 9 times stronger than the first column. Since the third column can be understood as a nano-porous material and the first column resembles the classical response, this example explains the importance of nano-materials and the need for computational homogenization to capture such size effects.

\subsubsection{Stress distribution for various strains}

The purpose of the last examples is to study the overall response of nano-porous material undergoing finite strains and compare it with the classical solution having no surface effects. Figures 9 and 10 illustrate the stress distributions on the RVE for two load cases of simple extension and simple shear, respectively. All simulations are carried out for $\xi=1 \%, \xi=$ $10 \%, \xi=20 \%$ and $\xi=40 \%$ with macro deformation gradients (31). 


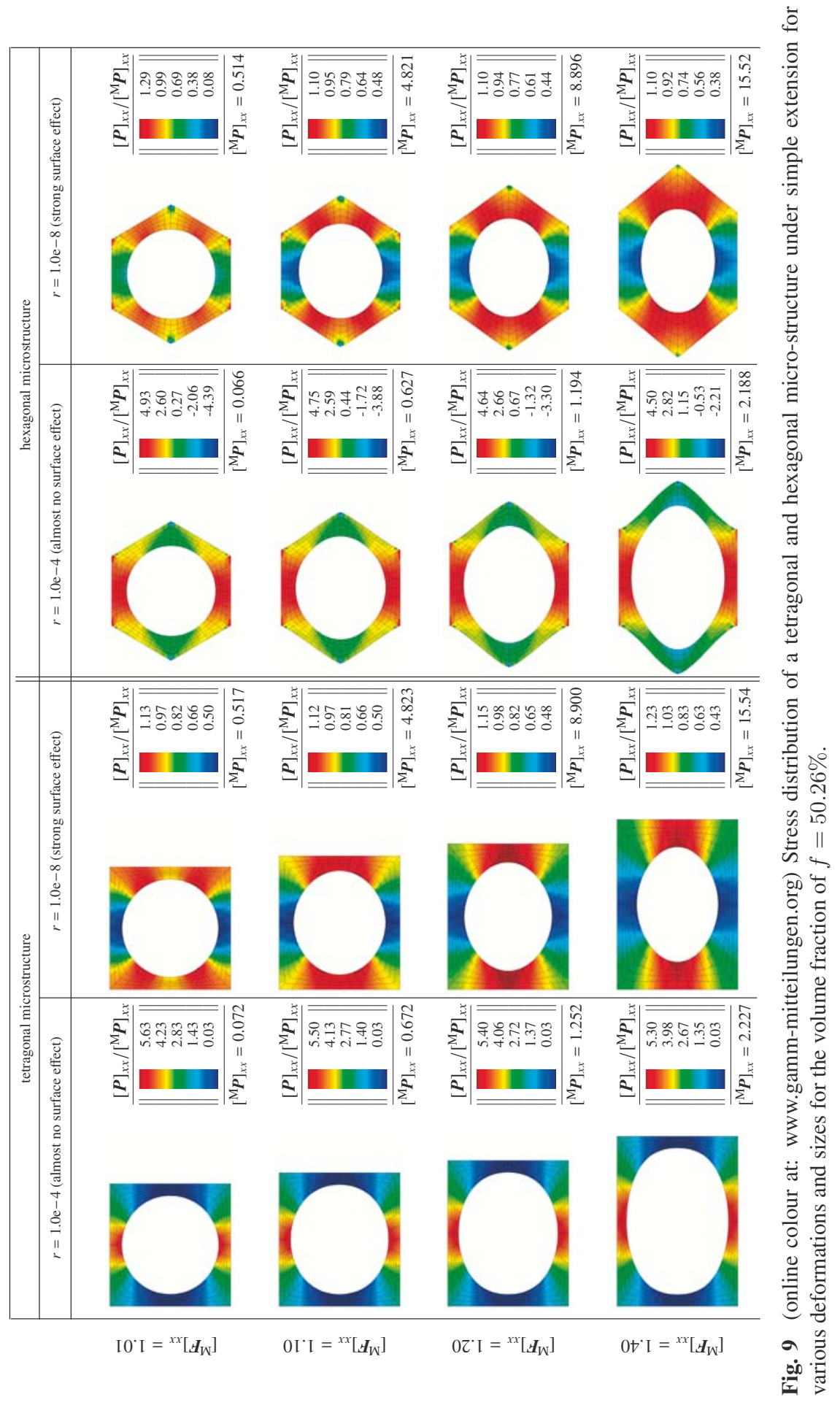




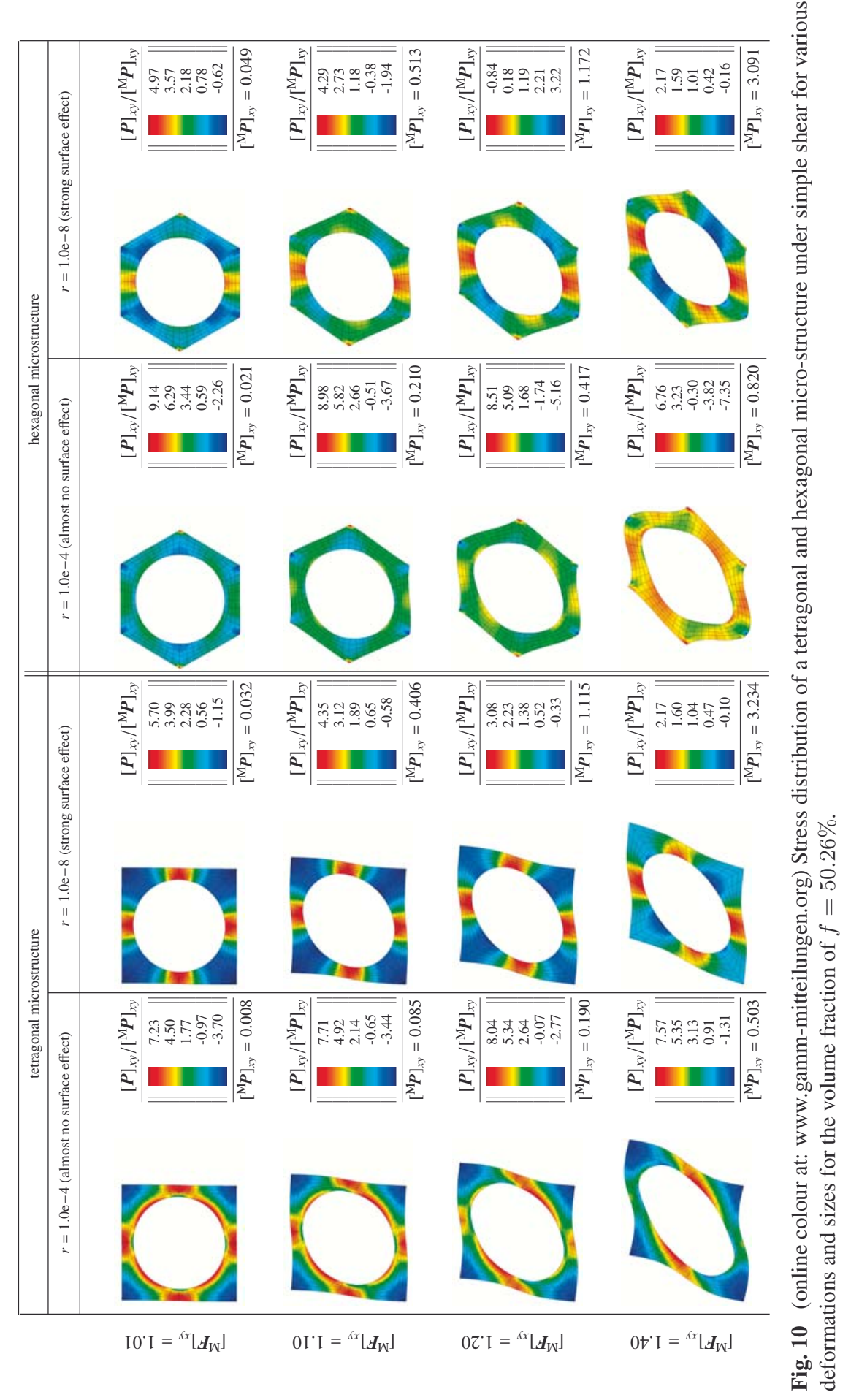


These results prove the robustness of the proposed framework at finite strains. For all the simulations the asymptotically quadratic rate of convergence associated with the NewtonRaphson scheme has been observed. The volume fraction of $f=50.26 \%$ is chosen for all the simulations since, from the previous examples, we know that at higher volume fractions a more pronounced surface effect is observed. Similar to the previous examples, the results show that nano-porous materials show a much stronger response compared to the classical homogenization. Also, increasing the deformation for a given column shows an increasing overall stress as expected, and importantly this trend is non-linear. For instance, although the deformation in the last row is 40 times the deformation in the first row, the macro stress of the last row is not 40 times the macro stress of the first row. In fact, for the simple extension load case the macro stress of the last row is around 30 times larger than the first row, but for the simple shear load case the macro stress of the last row is around 100 times larger the macro stress of the first row.

\section{Conclusion}

The effective properties of heterogeneous materials can be estimated from the response of the underlying microstructures using the computational homogenization procedure. The core idea of computational homogenization is to compute implicitly the unknown material response at the macroscale via homogenizing the response at the microscale where the constitutive behavior of each constituent is known. It is well-known that the size of the microstructure influences the material response, this is often termed as size-effect phenomenon. Nevertheless, standard first-order computational homogenization cannot capture the size-effect since the microscale lacks a length scale. In order to introduce a length scale to the computational homogenization, Kouznetsova et al. [35] have introduced higher gradients. Here, we propose a different strategy by including the surface energies at the microscale. This framework is physically motivated by the fact that the surface energies of the pores are no longer negligible at small scales. Davydov et al. [59] have recently shown an excellent agreement between this enhanced computational homogenization and atomistic simulations. Clearly, a distinct advantage of this enhanced model over its atomistic counterpart is the greatly increased computational efficiency. Also, this framework is computationally straightforward as it is intrinsically still a first-order homogenization and does not involve higher gradients.

One advantage of this framework is that it furnishes a more accurate solution compared to the analytical estimate for this problem. Furthermore, the analytical approximation is limited by the assumption of small strains linear elasticity whereas the current framework is developed for finite strains. It is shown that there exists an upper bound for the response of nano-porous materials which restricts the notion of "smaller is stronger"and has important applications in fabrication of nano-materials. This contributions deals with academic examples and parametric studies to better understand the problem and the methodology. Equipped with this framework, our immediate objective is to focus on specific physical applications and quantitatively assess the validity of this scheme and its advantages over analytical estimates. Further extensions of this work include studying nano-porous materials with negative surface parameters and perform instability analysis on the overall material response.

In summary, this manuscript presents our attempt to elucidate first-order computational homogenization accounting for size effects via the consideration of surfaces at the microscale. 
This allows one to precisely predict the overall response of nano-porous materials. We believe that this generic framework is broadly applicable to enhance our understanding of the behavior of materials with a large variety of applications to nano-materials.

\section{Acknowledgements}

The support of this work by the ERC Advanced Grant MOCOPOLY is gratefully acknowledged. AM thanks the support provided by the National Research Foundation of South Africa through the South African Research Chair in Computational Mechanics. CL also, acknowledges the support of Samsung Electronics.

\section{A Identities useful the generalized averaging theorems}

\section{A.1 Proof of identity 1:}

In order to prove the identity

$$
\int_{\partial \mathcal{B}_{0}} \boldsymbol{N} \otimes \boldsymbol{X} \mathrm{d} A=\mathscr{V}_{0} \boldsymbol{I}
$$

for the particular case of porous media, we first note that $\partial \mathcal{B}_{0}$ indicates the external boundary of the RVE. Due to opposite orientations of the external and internal surfaces from Fig. 2 and using the gradient theorem,

$$
\begin{aligned}
\int_{\partial \mathcal{B}_{0}} \boldsymbol{N} \otimes \boldsymbol{X} \mathrm{d} A & =\int_{\mathcal{B}_{0}}[\operatorname{Grad} \boldsymbol{X}]^{\mathrm{t}} \mathrm{d} V-\int_{\mathcal{S}_{0}} \boldsymbol{N} \otimes \widehat{\boldsymbol{X}} \mathrm{d} A \\
& =\int_{\mathcal{B}_{0}}[\operatorname{Grad} \boldsymbol{X}]^{\mathrm{t}} \mathrm{d} V+\int_{\mathcal{V}_{0}}[\operatorname{Grad} \boldsymbol{X}]^{\mathrm{t}} \mathrm{d} V \\
& =\int_{\mathcal{B}_{0}} \boldsymbol{I} \mathrm{d} V+\int_{\mathcal{V}_{0}} \boldsymbol{I} \mathrm{d} V \\
& =\boldsymbol{I}\left[\int_{\mathcal{B}_{0}} \mathrm{~d} V+\int_{\mathcal{V}_{0}} \mathrm{~d} V\right] \\
& =\mathscr{V}_{0} \boldsymbol{I} .
\end{aligned}
$$

\section{A.2 Proof of identity 2:}

In order to prove the identity

$$
\langle\boldsymbol{P}\rangle=\frac{1}{\mathscr{V}_{0}} \int_{\partial \mathcal{B}_{0}} \boldsymbol{t}_{0}^{\mathrm{p}} \otimes \boldsymbol{X} \mathrm{d} A+\frac{1}{\mathscr{V}_{0}} \int_{\mathcal{S}_{0}} \boldsymbol{t}_{0}^{\mathrm{p}} \otimes \widehat{\boldsymbol{X}} \mathrm{d} A-\frac{1}{\mathscr{V}_{0}} \int_{\mathcal{S}_{0}} \widehat{\boldsymbol{P}} \mathrm{d} A,
$$

we start from the relation

$$
\langle\boldsymbol{P}\rangle=\frac{1}{\mathscr{V}_{0}} \int_{\mathcal{B}_{0}} \boldsymbol{P} \mathrm{d} V+\frac{1}{\mathscr{V}_{0}} \int_{\mathcal{V}_{0}} \boldsymbol{P} \mathrm{d} V .
$$


Since we consider only porous materials, the second integral vanishes as the stresses are zero within the pores.

$$
\langle\boldsymbol{P}\rangle=\frac{1}{\mathscr{V}_{0}} \int_{\mathcal{B}_{0}} \boldsymbol{P} \mathrm{d} V=\frac{1}{\mathscr{V}_{0}} \int_{\mathcal{B}_{0}} \boldsymbol{P} \cdot \boldsymbol{I} \mathrm{d} V=\frac{1}{\mathscr{V}_{0}} \int_{\mathcal{B}_{0}} \boldsymbol{P} \cdot \operatorname{Grad} \boldsymbol{X} \mathrm{d} V
$$

using the identity $\boldsymbol{P} \cdot \operatorname{Grad} \boldsymbol{X}=\operatorname{Div}(\boldsymbol{P} \bar{\otimes} \boldsymbol{X})-\operatorname{Div} \boldsymbol{P} \otimes \boldsymbol{X}$ with $[\boldsymbol{P} \bar{\otimes} \boldsymbol{X}]_{i j k}=[\boldsymbol{P}]_{i k}[\boldsymbol{X}]_{j}$ and since $\operatorname{Div} \boldsymbol{P}=\mathbf{0}$ inside $\mathcal{B}_{0}$,

$$
=\frac{1}{\mathscr{V}_{0}} \int_{\mathcal{B}_{0}} \operatorname{Div}(\boldsymbol{P} \bar{\otimes} \boldsymbol{X}) \mathrm{d} V
$$

applying the divergence theorem and considering the orientations of the surface normals,

$$
\begin{aligned}
& =\frac{1}{\mathscr{V}_{0}} \int_{\partial \mathcal{B}_{0}}[\boldsymbol{P} \bar{\otimes}] \cdot \boldsymbol{N} \mathrm{d} A+\frac{1}{\mathscr{V}_{0}} \int_{\mathcal{S}_{0}}[\boldsymbol{P} \bar{\otimes} \widehat{\boldsymbol{X}}] \cdot \boldsymbol{N} \mathrm{d} A \\
& =\frac{1}{\mathscr{V}_{0}} \int_{\partial \mathcal{B}_{0}} \boldsymbol{P} \cdot \boldsymbol{N} \otimes \boldsymbol{X} \mathrm{d} A+\frac{1}{\mathscr{V}_{0}} \int_{\mathcal{S}_{0}} \boldsymbol{P} \cdot \boldsymbol{N} \otimes \widehat{\boldsymbol{X}} \mathrm{d} A
\end{aligned}
$$

from balance equations $(6)_{1}$ and $(6)_{2}$,

$$
=\frac{1}{\mathscr{V}_{0}} \int_{\partial \mathcal{B}_{0}} \boldsymbol{t}_{0}^{\mathrm{p}} \otimes \boldsymbol{X} \mathrm{d} A+\frac{1}{\mathscr{V}_{0}} \int_{\mathcal{S}_{0}} \boldsymbol{t}_{0}^{\mathrm{p}} \otimes \widehat{\boldsymbol{X}} \mathrm{d} A+\frac{1}{\mathscr{V}_{0}} \int_{\mathcal{S}_{0}} \widehat{\operatorname{Div}} \widehat{\boldsymbol{P}} \otimes \widehat{\boldsymbol{X}} \mathrm{d} A
$$

using the relation $\widehat{\operatorname{Div}} \widehat{\boldsymbol{P}} \otimes \widehat{\boldsymbol{X}}=\widehat{\operatorname{Div}}(\widehat{\boldsymbol{P}} \bar{\otimes} \widehat{\boldsymbol{X}})-\widehat{\boldsymbol{P}} \cdot \widehat{\operatorname{Grad}} \widehat{\boldsymbol{X}}=\widehat{\operatorname{Div}}(\widehat{\boldsymbol{P}} \bar{\otimes} \widehat{\boldsymbol{X}})-\widehat{\boldsymbol{P}} \cdot \widehat{\boldsymbol{I}}=$ $\widehat{\operatorname{Div}}(\widehat{\boldsymbol{P}} \bar{\otimes} \widehat{\boldsymbol{X}})-\widehat{\boldsymbol{P}}$

$$
\begin{aligned}
=\frac{1}{\mathscr{V}_{0}} \int_{\partial_{\mathcal{B}_{0}}} \boldsymbol{t}_{0}^{\mathrm{p}} \otimes \boldsymbol{X} \mathrm{d} A+\frac{1}{\mathscr{V}_{0}} \int_{\mathcal{S}_{0}} \boldsymbol{t}_{0}^{\mathrm{p}} \otimes \widehat{\boldsymbol{X}} \mathrm{d} A \\
\quad-\frac{1}{\mathscr{V}_{0}} \int_{\mathcal{S}_{0}} \widehat{\boldsymbol{P}} \mathrm{d} A+\frac{1}{\mathscr{V}_{0}} \int_{\mathcal{S}_{0}} \widehat{\operatorname{Div}}(\widehat{\boldsymbol{P}} \bar{\otimes} \widehat{\boldsymbol{X}}) \mathrm{d} A
\end{aligned}
$$

with the aids of the surface divergence theorem and denoting the surface curvature as $\widehat{\kappa}$ the last term is rewritten,

$$
\begin{aligned}
& =\frac{1}{\mathscr{V}_{0}} \int_{\partial \mathcal{B}_{0}} \boldsymbol{t}_{0}^{\mathrm{p}} \otimes \boldsymbol{X} \mathrm{d} A+\frac{1}{\mathscr{V}_{0}} \int_{\mathcal{S}_{0}} \boldsymbol{t}_{0}^{\mathrm{p}} \otimes \widehat{\boldsymbol{X}} \mathrm{d} A \\
& \quad-\frac{1}{\mathscr{V}_{0}} \int_{\mathcal{S}_{0}} \widehat{\boldsymbol{P}} \mathrm{d} A+\frac{1}{\mathscr{V}_{0}} \int_{\mathcal{S}_{0}} \widehat{\kappa} \widehat{\boldsymbol{P}} \cdot \boldsymbol{N} \otimes \widehat{\boldsymbol{X}} \mathrm{d} A+\frac{1}{\mathscr{V}_{0}} \int_{\partial \mathcal{S}_{0}} \widehat{\boldsymbol{P}} \cdot \widehat{\boldsymbol{N}} \otimes \widehat{\boldsymbol{X}} \mathrm{d} L
\end{aligned}
$$

the last term vanishes as we are considering closed surfaces entirely within the domain. the second last term vanishes due to the superficial characteristic of the surface Piola stress tensor and finally,

$$
=\frac{1}{\mathscr{V}_{0}} \int_{\partial \mathcal{B}_{0}} \boldsymbol{t}_{0}^{\mathrm{p}} \otimes \boldsymbol{X} \mathrm{d} A+\frac{1}{\mathscr{V}_{0}} \int_{\mathcal{S}_{0}} \boldsymbol{t}_{0}^{\mathrm{p}} \otimes \widehat{\boldsymbol{X}} \mathrm{d} A-\frac{1}{\mathscr{V}_{0}} \int_{\mathcal{S}_{0}} \widehat{\boldsymbol{P}} \mathrm{d} A
$$


or alternatively,

$$
\langle\boldsymbol{P}\rangle_{\mathcal{B}_{0}}+\langle\widehat{\boldsymbol{P}}\rangle_{\mathcal{S}_{0}}=\frac{1}{\mathscr{V}} \int_{\partial \mathcal{B}_{0}} \boldsymbol{t}_{0}^{\mathrm{p}} \otimes \boldsymbol{X} \mathrm{d} A+\frac{1}{\mathscr{V}_{0}} \int_{\mathcal{S}_{0}} \boldsymbol{t}_{0}^{\mathrm{p}} \otimes \widehat{\boldsymbol{X}} \mathrm{d} A
$$

\section{A.3 Generalized Hill's Lemma}

In order to prove the identity

$$
\begin{aligned}
\langle\boldsymbol{P}: \delta \boldsymbol{F}\rangle+\langle\widehat{\boldsymbol{P}}: \delta \widehat{\boldsymbol{F}}\rangle_{\mathcal{S}_{0}} & -{ }^{\mathrm{M}} \boldsymbol{P}: \delta^{\mathrm{M}} \boldsymbol{F} \\
& =\frac{1}{\mathscr{V}_{0}} \int_{\partial \mathcal{B}_{0}}\left[\delta \boldsymbol{\varphi}-\delta^{\mathrm{M}} \boldsymbol{F} \cdot \boldsymbol{X}\right] \cdot\left[\boldsymbol{t}_{0}^{\mathrm{p}}-{ }^{\mathrm{M}} \boldsymbol{P} \cdot \boldsymbol{N}\right] \mathrm{d} A,
\end{aligned}
$$

the right-hand side is expanded and manipulated until it eventually results in the left-hand side. Since equality operator is used exclusively, all the steps are also valid in the reverse order. First, we use the relation $\boldsymbol{t}_{0}^{\mathrm{p}}=\boldsymbol{P} \cdot \boldsymbol{N}$ on $\partial \mathcal{B}_{0}$ and expand the right-hand side.

$$
\begin{aligned}
\int_{\partial \mathcal{B}_{0}}\left[\delta \boldsymbol{\varphi}-\delta^{\mathrm{M}} \boldsymbol{F} \cdot \boldsymbol{X}\right] \cdot\left[\boldsymbol{t}_{0}^{\mathrm{p}}-{ }^{\mathrm{M}} \boldsymbol{P} \cdot \boldsymbol{N}\right] \mathrm{d} A \\
=\int_{\partial \mathcal{B}_{0}}\left[\delta \boldsymbol{\varphi}-\delta^{\mathrm{M}} \boldsymbol{F} \cdot \boldsymbol{X}\right] \cdot\left[\boldsymbol{P} \cdot \boldsymbol{N}-{ }^{\mathrm{M}} \boldsymbol{P} \cdot \boldsymbol{N}\right] \mathrm{d} A \\
=\int_{\partial \mathcal{B}_{0}} \delta \boldsymbol{\varphi} \cdot[\boldsymbol{P} \cdot \boldsymbol{N}] \mathrm{d} A-\int_{\partial \mathcal{B}_{0}} \delta \boldsymbol{\varphi} \cdot\left[{ }^{\mathrm{M}} \boldsymbol{P} \cdot \boldsymbol{N}\right] \mathrm{d} A \\
\quad-\int_{\partial \mathcal{B}_{0}}\left[\delta^{\mathrm{M}} \boldsymbol{F} \cdot \boldsymbol{X}\right] \cdot \boldsymbol{P} \cdot \boldsymbol{N} \mathrm{d} A+\int_{\partial \mathcal{B}_{0}}\left[\delta^{\mathrm{M}} \boldsymbol{F} \cdot \boldsymbol{X}\right] \cdot{ }^{\mathrm{M}} \boldsymbol{P} \cdot \boldsymbol{N} \mathrm{d} A \\
=\int_{\partial \mathcal{B}_{0}}[\delta \boldsymbol{\varphi} \cdot \boldsymbol{P}] \cdot \boldsymbol{N} \mathrm{d} A-\int_{\partial \mathcal{B}_{0}}{ }^{\mathrm{M}} \boldsymbol{P}:[\delta \boldsymbol{\varphi} \otimes \boldsymbol{N}] \mathrm{d} A \\
\quad-\int_{\partial \mathcal{B}_{0}} \delta^{\mathrm{M}} \boldsymbol{F}:[[\boldsymbol{P} \cdot \boldsymbol{N}] \otimes \boldsymbol{X}] \mathrm{d} A+\int_{\partial \mathcal{B}_{0}}\left[\delta^{\mathrm{M}} \boldsymbol{F}^{\mathrm{t}} \cdot{ }^{\mathrm{M}} \boldsymbol{P}\right]:[\boldsymbol{X} \otimes \boldsymbol{N}] \mathrm{d} A
\end{aligned}
$$

Using the generic relation $\int_{\partial \mathcal{B}_{0}}\{\bullet\} \cdot N \mathrm{~d} A=\int_{\mathcal{B}_{0}} \operatorname{Div}\{\bullet\} \mathrm{d} V-\int_{\mathcal{S}_{0}}\{\bullet\} \cdot N \mathrm{~d} A$ on the first integral, with $\{\bullet\}$ denoting any arbitrary vector or tensor quantity, and taking the constants out of the integral operators,

$$
\begin{array}{r}
=\int_{\mathcal{B}_{0}} \operatorname{Div}(\delta \boldsymbol{\varphi} \cdot \boldsymbol{P}) \mathrm{d} V-\int_{\mathcal{S}_{0}}[\delta \widehat{\boldsymbol{\varphi}} \cdot \boldsymbol{P}] \cdot \boldsymbol{N} \mathrm{d} A-{ }^{\mathrm{M}} \boldsymbol{P}: \int_{\partial \mathcal{B}_{0}} \delta \boldsymbol{\varphi} \otimes \boldsymbol{N} \mathrm{d} A \\
\quad-\delta^{\mathrm{M}} \boldsymbol{F}: \int_{\partial \mathcal{B}_{0}}[[\boldsymbol{P} \cdot \boldsymbol{N}] \otimes \boldsymbol{X}] \mathrm{d} A+\left[\delta^{\mathrm{M}} \boldsymbol{F}^{\mathrm{t}} \cdot{ }^{\mathrm{M}} \boldsymbol{P}\right]: \int_{\partial \mathcal{B}_{0}} \boldsymbol{X} \otimes \boldsymbol{N} \mathrm{d} A
\end{array}
$$


The first integral is simplified with the relation $\operatorname{Div}(\delta \boldsymbol{\varphi} \cdot \boldsymbol{P})=\delta \boldsymbol{\varphi} \cdot \operatorname{Div} \boldsymbol{P}+\boldsymbol{P}: \operatorname{Grad} \delta \boldsymbol{\varphi}=$ $\boldsymbol{P}: \delta \boldsymbol{F}$ since $\operatorname{Div} \boldsymbol{P}=\mathbf{0}$ inside $\mathcal{B}_{0}$. The relation $\widehat{\operatorname{Div}} \widehat{\boldsymbol{P}}=\boldsymbol{P} \cdot \boldsymbol{N}$ is applied on the second integral. The third integral is rewritten motivated by the definition of macro deformation gradient (20) as $\int_{\partial \mathcal{B}_{0}} \delta \boldsymbol{\varphi} \otimes \boldsymbol{N} \mathrm{d} A=\mathscr{V}_{0} \delta^{\mathrm{M}} \boldsymbol{F}$. The fourth integral is indeed the first term on the right-hand side of the identity 2 since $\boldsymbol{t}_{0}^{\mathrm{p}}=\boldsymbol{P} \cdot \boldsymbol{N}$ on $\partial \mathcal{B}_{0}$ and therefore it can be expressed as $\int_{\partial \mathcal{B}_{0}}[[\boldsymbol{P} \cdot \boldsymbol{N}] \otimes \boldsymbol{X}] \mathrm{d} A=\mathscr{V}_{0}\left[\langle\boldsymbol{P}\rangle+\langle\widehat{\boldsymbol{P}}\rangle_{\mathcal{S}_{0}}\right]$ as $\boldsymbol{t}_{0}^{\mathrm{p}}=\mathbf{0}$ on $\mathcal{S}_{0}$. The last integral simplifies using the identity (12) in its transposed format as $\int_{\partial \mathcal{B}_{0}} \boldsymbol{X} \otimes \boldsymbol{N} \mathrm{d} A=\mathscr{V}_{0} \boldsymbol{I}$.

$$
\begin{array}{r}
=\int_{\mathcal{B}_{0}} \boldsymbol{P}: \delta \boldsymbol{F} \mathrm{d} V-\int_{\mathcal{S}_{0}} \delta \widehat{\boldsymbol{\varphi}} \cdot \widehat{\operatorname{Div}} \widehat{\boldsymbol{P}} \mathrm{d} A-\mathscr{V}_{0}{ }^{\mathrm{M}} \boldsymbol{P}: \delta^{\mathrm{M}} \boldsymbol{F} \\
\quad-\mathscr{V}_{0} \delta^{\mathrm{M}} \boldsymbol{F}:\left[\langle\boldsymbol{P}\rangle+\langle\widehat{\boldsymbol{P}}\rangle_{\mathcal{S}_{0}}\right]+\left[\delta^{\mathrm{M}} \boldsymbol{F}^{\mathrm{t}} \cdot{ }^{\mathrm{M}} \boldsymbol{P}\right]:\left[\mathscr{V}_{0} \boldsymbol{I}\right]
\end{array}
$$

The second integral is expanded using the relation $\delta \widehat{\varphi} \cdot \widehat{\operatorname{Div}} \widehat{\boldsymbol{P}}=\widehat{\operatorname{Div}}(\delta \widehat{\boldsymbol{\varphi}} \cdot \widehat{\boldsymbol{P}})-\widehat{\boldsymbol{P}}: \widehat{\operatorname{Grad}} \delta \widehat{\boldsymbol{\varphi}}$.

$$
\begin{aligned}
=\mathscr{V}_{0} & \langle\boldsymbol{P}: \delta \boldsymbol{F}\rangle_{\mathcal{B}_{0}}-\int_{\mathcal{S}_{0}} \widehat{\operatorname{Div}}(\delta \widehat{\boldsymbol{\varphi}} \cdot \widehat{\boldsymbol{P}}) \mathrm{d} A+\int_{\mathcal{S}_{0}} \widehat{\boldsymbol{P}}: \widehat{\operatorname{Grad}} \delta \widehat{\boldsymbol{\varphi}} \mathrm{d} A-\mathscr{V}_{0}{ }^{\mathrm{M}} \boldsymbol{P}: \delta^{\mathrm{M}} \boldsymbol{F} \\
& -\mathscr{V}_{0}\langle\boldsymbol{P}\rangle: \delta^{\mathrm{M}} \boldsymbol{F}-\mathscr{V}_{0}\langle\widehat{\boldsymbol{P}}\rangle_{\mathcal{S}_{0}}: \delta^{\mathrm{M}} \boldsymbol{F}+\mathscr{V}_{0}\left[\delta^{\mathrm{M}} \boldsymbol{F}^{\mathrm{t}} \cdot{ }^{\mathrm{M}} \boldsymbol{P}\right]: \boldsymbol{I}
\end{aligned}
$$

The second integral can be further expanded using the surface divergence theorem and eventually vanishes due to the superficiality of the surface Piola stress and considering closed surfaces similar to the proof of the identity (16).

$$
\begin{aligned}
=\mathscr{V}_{0}\langle\boldsymbol{P}: \delta \boldsymbol{F}\rangle_{\mathcal{B}_{0}}+\mathscr{V}_{0}\langle\widehat{\boldsymbol{P}}: \delta \widehat{\boldsymbol{F}}\rangle_{\mathcal{S}_{0}}-\mathscr{V}_{0}{ }^{\mathrm{M}} \boldsymbol{P}: \delta^{\mathrm{M}} \boldsymbol{F} \\
-\underbrace{\left[\mathscr{V}_{0}\langle\boldsymbol{P}\rangle: \delta^{\mathrm{M}} \boldsymbol{F}+\mathscr{V}_{0}\langle\widehat{\boldsymbol{P}}\rangle_{\mathcal{S}_{0}}: \delta^{\mathrm{M}} \boldsymbol{F}\right]}_{\mathscr{V}_{0}\left[\langle\boldsymbol{P}\rangle+\langle\widehat{\boldsymbol{P}}\rangle_{\mathcal{S}_{0}}\right]: \delta^{\mathrm{M}} \boldsymbol{F}}+\mathscr{V}_{0}{ }^{\mathrm{M}} \boldsymbol{P}: \delta^{\mathrm{M}} \boldsymbol{F}
\end{aligned}
$$

The third term vanishes together with the last term. Also, from the definition of the macro Piola stress (18) we have ${ }^{\mathrm{M}} \boldsymbol{P}=\langle\boldsymbol{P}\rangle_{\mathcal{B}_{0}}+\langle\widehat{\boldsymbol{P}}\rangle_{\mathcal{S}_{0}}=\langle\boldsymbol{P}\rangle+\langle\widehat{\boldsymbol{P}}\rangle_{\mathcal{S}_{0}}$ and therefore,

$$
=\mathscr{V}_{0}\left[\langle\boldsymbol{P}: \delta \boldsymbol{F}\rangle_{\mathcal{B}_{0}}+\langle\widehat{\boldsymbol{P}}: \delta \widehat{\boldsymbol{F}}\rangle_{\mathcal{S}_{0}}-{ }^{\mathrm{M}} \boldsymbol{P}: \delta^{\mathrm{M}} \boldsymbol{F}\right]
$$

\section{References}

[1] S. Iijima, Nature 354, 56-58 (1991).

[2] H. Masuda, H. Yamada, M. Satoh, H. Asoh, M. Nakao, and T. Tamamura, Appl. Phys. Lett. 71(19), 2770 (1997).

[3] E. W. Wong, Science 277(5334), 1971-1975 (1997).

[4] A. M. Morales, Science 279(5348), 208-211 (1998).

[5] K. S. Kumar, H. Van Swygenhoven, and S. Suresh, Acta Mater. 51(19), 5743-5774 (2003). 
[6] X. Y. Kong and Z. L. Wang, Nano Lett. 3(12), 1625-1631 (2003).

[7] V. Ramaswamy, W. D. Nix, and B. M. Clemens, Scr. Mater. 50(6), 711-715 (2004).

[8] H. L. Duan, J. Wang, and B. L. Karihaloo, Adv. Appl. Mech. 42, 1-68 (2009).

[9] A. Javili, A. Mcbride, and P. Steinmann, Appl. Mech. Rev. 65, 010802 (2013).

[10] M.E. Gurtin and A. Ian Murdoch, Arch. Ration. Mech. Anal. 57(1941), 291-323 (1975).

[11] L. Scriven, Chem. Eng. Sci. 12(2), 98-108 (1960).

[12] G. P. Moeckel, Arch. Ration. Mech. Anal. 57, 255-280 (1975).

[13] A. I. Murdoch, Q. J. Mech. Appl. Math. 29(3), 245-275 (1976).

[14] N. Daher and G. A. Maugin, Acta Mech. 60, 217-240 (1986).

[15] F. Dell'Isola and A. Romano, Int. J. Eng. Sci. 25, 1459-1468 (1987).

[16] D. J. Steigmann and R. W. Ogden, Proc. R. Soc. A 455, 437-474 (1999).

[17] A. Javili and P. Steinmann, Int. J. Solids Struct. 47, 3245-3253 (2010).

[18] G. Kaptay, J. Mater. Sci. 40, 2125-2131 (2005).

[19] G. Wei, Y. Shouwen, and H. Ganyun, Nanotechnology 17(4), 1118-1122 (2006).

[20] H. S. Park and P. A. Klein, Comput. Methods Appl. Mech. Eng. 197, 3249-3260 (2008).

[21] J. Yvonnet, H. L. Quang, and Q. C. He, Comput. Mech. 42, 119-131 (2008).

[22] A. Javili and P. Steinmann, Comput. Methods Appl. Mech. Eng. 198, 2198-2208 (2009).

[23] A. Javili and P. Steinmann, Comput. Methods Appl. Mech. Eng. 199, 755-765 (2010).

[24] A. Javili, A. McBride, P. Steinmann, and B. D. Reddy, Comput. Mech. 54, 745-762 (2014).

[25] R. Hill, J. Mech. Phys. Solids 11, 357-372 (1963).

[26] R. Hill and J. Rice, J. Mech. Phys. Solids 20, 401-413 (1972).

[27] P. M. Suquet, Lecture Notes in Physics 272, 193-278 (1987).

[28] M. Guedes and N. Kikuchi, Comput. Methods Appl. Mech. Eng. 83, 143-198 (1990).

[29] K. Terada and N. Kikuchi, American Soc. Mech. Eng., Appl. Mech. Div., AMD 212, 1-16 (1995).

[30] R. Smit, W. Brekelmans, and H. Meijer, Comput. Methods Appl. Mech. Eng. 155, 181-192 (1998).

[31] C. Miehe, J. Schröder, and J. Schotte, Comput. Methods Appl. Mech. Eng. 171, 387-418 (1999).

[32] J. C. Michel, H. Moulinec, and P. Suquet, Comput. Methods Appl. Mech. Eng. 172, 109-143 (1999).

[33] F. Feyel and J. L. Chaboche, Comput. Methods Appl. Mech. Eng. 183, 309-330 (2000).

[34] V. Kouznetsova, W. a. M. Brekelmans, and F. P. T. Baaijens, Comput. Mech. 27, 37-48 (2001).

[35] V. Kouznetsova, M. G. D. Geers, and W. a. M. Brekelmans, Int. J. Numer. Methods Eng. 54, 1235-1260 (2002).

[36] T. I. Zohdi and P. Wriggers, Arch. Comput. Methods Eng. 8, 131-228 (2001).

[37] C. Miehe, Int. J. Numer. Methods Eng. 55, 1285-1322 (2002).

[38] C. Miehe and A. Koch, Arch. Appl. Mech. 72, 300-317 (2002).

[39] I. Temizer and P. Wriggers, Comput. Methods Appl. Mech. Eng. 198(3-4), 495-510 (2008).

[40] P. Kanouté, D. P. Boso, J. L. Chaboche, and B.a. Schrefler, Arch. Comput. Methods Eng. 16, 31-75 (2009).

[41] F. Larsson, K. Runesson, and F. Su, Int. J. Numer. Anal. Meth. Geomech. 34, 1431-1458 (2010).

[42] C. Linder, M. Tkachuk, and C. Miehe, J. Mech. Phys. Solids 59(10), 2134-2156 (2011).

[43] A. McBride, J. Mergheim, A. Javili, P. Steinmann, and S. Bargmann, J. Mech. Phys. Solids 60(6), 1221-1239 (2012).

[44] M. Tkachuk and C. Linder, Philos. Mag. 92(22), 2779-2808 (2012).

[45] D. M. Kochmann and G. N. Venturini, Smart Mater. Struct. 22, 084004 (2013).

[46] A. Raina and C. Linder, J. Mech. Phys. Solids 65(1), 12-34 (2014).

[47] B. S. Mercer, K. K. Mandadapu, and P. Papadopoulos, Comput. Methods Appl. Mech. Eng. 286, 268-292 (2015).

[48] T. Miloh and Y. Benveniste, Proc. R. Soc. A Math. Phys. Eng. Sci. 455(1987), 2687-2706 (1999).

[49] P. Sharma and S. Ganti, J. Appl. Mech. 71, 663-671 (2004).

[50] H. L. Duan, J. Wang, Z. P. Huang, and B. L. Karihaloo, J. Mech. Phys. Solids 53(7), 1574-1596 (2005).

[51] H. L. Duan, J. Wang, B. L. Karihaloo, and Z. P. Huang, Acta Mater. 54, 2983-2990 (2006). 
[52] Y. Benveniste, Int. J. Eng. Sci. 72, 140-154 (2013).

[53] A. Javili, A. Mcbride, J. Mergheim, P. Steinmann, and U. Schmidt, Int. J. Solids Struct. 50, 2561-2572 (2013).

[54] Z. F. Khisaeva, and M. Ostoja-Starzewski, J. Elast. 85, 153-173 (2006).

[55] A. Javili, F. Dell'Isola, and P. Steinmann, J. Mech. Phys. Solids 61, 2381-2401 (2013).

[56] C. Miehe, Comput. Methods Appl. Mech. Eng. 192, 559-591 (2003).

[57] P. Steinmann, Journal of the Mechanics and Physics of Solids 56, 772-800 (2008).

[58] V. B. Shenoy, Phys. Rev. B - Condens. Matter Mater. Phys. 71(9), 1-11 (2005).

[59] D. Davydov, A. Javili, and P. Steinmann, Comput. Mater. Sci. 69, 510-519 (2013).

[60] A. Javili, A. McBride, P. Steinmann, and B. Reddy, Philos. Mag. 92, 3540-3563 (2012).

[61] G. Chatzigeorgiou, A. Javili, and P. Steinmann, Math. Mech. Solids (2015).

[62] Z. Hashin and B. W. Rosen, J. Appl. Mech. Trans. ASME 31(63), 223-232 (1964).

[63] R. M. Christensen, Mechanics of composite materials (Dover, 1979). 